\title{
Partial twisting for scalar mesons
}

\section{Dimitri Agadjanov, ${ }^{a, b}$ Ulf-G. Meißner ${ }^{a, c}$ and Akaki Rusetsky ${ }^{a}$}

${ }^{a}$ Helmholtz-Institut für Strahlen- und Kernphysik (Theorie) and Bethe Center for Theoretical Physics, Universität Bonn, Nussallee 12, D-53115 Bonn, Germany

${ }^{b}$ St. Andrew the First-Called Georgian University of the Patriarchate of Georgia, Chavchavadze Ave. 53a, 0162, Tbilisi, Georgia

${ }^{c}$ Institute for Advanced Simulation (IAS-4), Institut für Kernphysik (IKP-3) and Jülich Center for Hadron Physics, Forschungszentrum Jülich, D-52425 Jülich, Germany

E-mail: dagadjanov@hiskp.uni-bonn.de, meissner@hiskp.uni-bonn.de, rusetsky@hiskp.uni-bonn.de

ABSTRACT: The possibility of imposing partially twisted boundary conditions is investigated for the scalar sector of lattice QCD. According to the commonly shared belief, the presence of quark-antiquark annihilation diagrams in the intermediate state generally hinders the use of the partial twisting. Using effective field theory techniques in a finite volume, and studying the scalar sector of QCD with total isospin $I=1$, we however demonstrate that partial twisting can still be performed, despite the fact that annihilation diagrams are present. The reason for this are delicate cancellations, which emerge due to the graded symmetry in partially quenched QCD with valence, sea and ghost quarks. The modified Lüscher equation in case of partial twisting is given.

KEYwords: Lattice QCD, Chiral Lagrangians

ARXIV EPRINT: 1310.7183 


\section{Contents}

1 Introduction $\quad 1$

2 The effective field theory framework 4

3 Symmetries of the potential $\quad 9$

4 Derivation of the partially twisted Lüscher equation 18

5 Meson mixing in the neutral sector $\quad 22$

6 Conclusions and outlook $\quad 26$

$\begin{array}{ll}\text { A Explicit form of the matrices } T_{j} \text { in eq. (2.13) } & 27\end{array}$

B Proof of eq. (5.15) 28

B.1 The structure of the matrix $\Lambda_{i \alpha} \quad 28$

B.2 The linear relations between the four-point functions 32

\section{Introduction}

Investigating the scalar sector of QCD in the region below and around $1 \mathrm{GeV}$ on the lattice enables one to gain important information about the low-energy behavior of strong interactions. A few groups have addressed this problem in the recent years (see, e.g., [17]). Note that carrying out simulations in the scalar sector is a very challenging task by itself as many of these states share the quantum numbers of the vacuum. In addition, it is known that the particles, whose properties are investigated in these simulations, are resonances. Consequently, in order to perform the extraction of their mass and width from the data, one has to apply the Lüscher approach [8] that implies carrying out simulations at different volumes, complicating further an already difficult problem. Moreover, in case of the $f_{0}(980)$ and $a_{0}(980)$ mesons, the analysis has to be done by using a coupled-channel Lüscher equation [9-11], which includes $\pi \pi / K \bar{K}$ and $\pi \eta / K \bar{K}$ channels for total isospin $I=0$ and $I=1$, respectively. The resonances are very close to the $K \bar{K}$ (inelastic) threshold, which has the unpleasant property of "masking" the avoided level crossing that serves as a signature of the presence of a resonance in a finite volume [9-11].

Here, one should also mention that the mass and width are not the only quantities one is interested in case of scalar resonances. The nature of these states is not well established in phenomenology and is being debated at present, with the arguments given in favor of their interpretation as tetraquark states (see, e.g., [12-15]), as $K \bar{K}$ molecules [16-19], or as 
a combination of a bare pole and the rescattering contribution [20, 21] (see also refs. [2224] for more information on this issue). In view of the conflicting interpretations, it is interesting to study the signatures of a possible exotic behavior, e.g., applying Weinberg's compositeness condition or the pole counting criterion (see, e.g., [25-34]), or investigating the quark mass dependence of the resonance pole position [9]. It is possible to "translate" all these criteria into the language of lattice QCD. However, testing them in the real simulations would require much more data at different volumes and at a much higher precision than it is at our disposal at present.

Summarizing all the facts above, it is legitimate to ask, whether - given our present capabilities - the extraction of the properties of scalar resonances on the lattice can be realistically done with a sufficient rigor and yield clean and unambiguous results in the nearest future.

In refs. [9-11] it has been pointed out that using twisted boundary conditions in lattice simulations [35-39] can provide an important advantage in the scalar meson sector (for applications of this method in other systems see, e.g., [40]). First and foremost, varying the twisting angle $\boldsymbol{\theta}$ can substitute for simulations at different volumes and provide data of energy levels, which should be fitted in order to determine the resonance pole position. Note that the same effect can be achieved by carrying out simulations at a non-zero total momentum. However, whereas the components of the lattice momentum are given by integer numbers in the units of $2 \pi / L$, where $L$ is the size of the finite box, the twisting angle can be varied continuously. Another advantage is provided by the fact that twisting allows one to effectively move the threshold away from the resonance pole location. In order to illustrate this, consider an example when the $s$-quark is twisted in the simulations, whereas $u$ and $d$ quarks still obey periodic boundary conditions [9-11]. Assume, in addition, that the system is in the center-of-mass $(\mathrm{CM})$ frame. In this example, the $K$ and $\bar{K}$ mesons in the $K \bar{K}$ intermediate state acquire 3-momenta, opposite in direction and having equal magnitude, proportional to $|\boldsymbol{\theta}|$. Hence, the energy of the ground state of the $K \bar{K}$ pair goes up, whereas the resonance, which corresponds to a true pole in the $S$-matrix, stays, by definition, at the same position. For the volumes, which are currently used in lattice simulations, the upward displacement of the $K \bar{K}$ threshold would be a large effect. Consequently, it could be expected that, fitting twisted lattice data, one would achieve a more accurate extraction of the resonance pole position than in the case of periodic boundary conditions, when the threshold and the resonance are very close. Note that this conjecture has been fully confirmed in refs. [10, 11] by performing fits to "synthetic" data sets.

There is, however, an important caveat in the arguments above. Imposing twisted boundary conditions in lattice simulations implies the calculation of gauge configurations anew. This is a very expensive enterprise. The majority of simulations up to day are done by applying the so-called partial twisting, i.e., twisting only the valence quarks and leaving the configurations the same. It can be proven (see, e.g. [38, 39]) that in many cases the results obtained by using partial and full twisting coincide up to exponentially suppressed terms. This happens when there are no annihilation diagrams, i.e., the diagrams where the valence quark-antiquark pair from the initial state can annihilate and a pair of the 


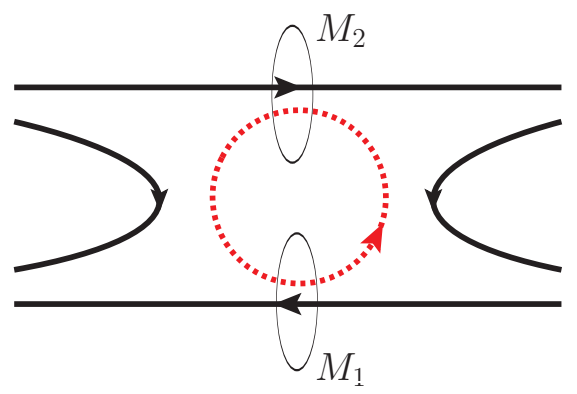

Figure 1. An example of an annihilation diagram in meson-meson scattering. The full and dashed lines denote valence and sea quarks, respectively. The intermediate state for this diagram consists of two mesons $M_{1}$ and $M_{2}$ with one valence and one sea quark.

sea quark-antiquark is produced, which obey a different boundary condition (see figure 1). However, it is easy to verify that, in case of scalar mesons, the annihilation diagrams do appear. Consequently, following the arguments of refs. [38, 39], one had to conclude that the partial twisting in this case is useless - one has either to perform a full twisting, or to give it up.

We consider this conclusion premature. One could look at the problem from a different point of view. It is definitely not possible to prove in general that in this case the partial and full twisting lead to the same result. Could one find a modified Lüscher equation, which corresponds to the case of partial twisting? Does this equation enable one to still extract the physically interesting information about the scattering $S$-matrix elements in the finite volume? If the answer to this question is yes, using partial twisting in lattice simulations can be justified.

In this paper we do not give a full-fledged solution of the problem. Rather, we have chosen to concentrate on one particular example, namely, the $a_{0}(980)$, which is an $S$-wave resonance with the isospin $I=1$, and solve this problem to the end. Possible mixing to other partial waves is neglected. The inclusion of higher partial waves forms a subject of a separate investigation which will be carried out in the future.

A brief outline of the method is as follows. It is well known that Lüscher's equation can be most easily derived by using non-relativistic EFT framework in a finite volume [41-43]. Twisting at the quark level can be straightforwardly implemented at the hadronic level: the hadrons acquire additional momenta, proportional to the twisting angle $\boldsymbol{\theta}$. The expression for the zeta-function in the Lüscher equation also changes in a well-defined way, whereas the non-relativistic potentials, which encode the short-range dynamics, are $\boldsymbol{\theta}$-independent. All this gives the Lüscher equation in case of twisted boundary conditions.

The case of partially twisted boundary conditions can be considered analogously. The spectrum of the effective theory now contains much more hadrons, consisting of valence, sea and ghost quarks (see, e.g., [44]). Boundary conditions for each hadron are determined by the boundary conditions on its constituents, so the $\boldsymbol{\theta}$-dependence of the zeta-functions, entering the Lüscher equation, is uniquely defined also in this case. The crucial observation, which enables one to arrive at a tractable form of the Lüscher equation, is that the 
symmetries, which are present in the theory in the infinite volume, relate the potentials in valence, sea and ghost sectors (the masses of valence, sea and ghost quarks are taken equal). It can be shown that the Lüscher equation can be reduced to the one that contains the potentials only in the physical (valence) sector and can thus be used to analyze the lattice data.

The layout of the paper is as follows. In section 2 we describe the effective field theory (EFT) framework for partially twisted QCD - first, in the infinite volume. In section 3 we discuss in detail the constraints imposed by the symmetries on the matrix elements of the effective non-relativistic potential. In doing this, we first neglect the neutral meson mixing beyond tree level. In section 4 the Lüscher equation in case of the partially twisted boundary conditions is derived. Possible applications in the simulations in the scalar sector are discussed. In section 5 we clear the remaining loopholes by discussing the mixing to all orders in this framework and show that the results are not affected. Finally, section 6 contains our conclusions and outlook.

\section{The effective field theory framework}

In order to obtain the spectrum, one usually studies the behavior of certain correlators at a large Euclidean time separation $t$ :

$$
C(t)=\left\langle\mathcal{O}(t) \mathcal{O}^{\dagger}(0)\right\rangle=\frac{1}{Z} \int \mathcal{D} U \mathcal{D} \psi \mathcal{D} \bar{\psi} \mathcal{O}(t) \mathcal{O}^{\dagger}(0) \exp \left\{-S_{G}-\int d^{4} x \bar{\psi}(\not D+m) \psi\right\}
$$

where $S_{G}$ stands for the gluon action functional, and $\mathcal{O}(t), \mathcal{O}^{\dagger}(t)$ are appropriate source/sink operators, which have a non-zero overlap with the physical states of interest. At this stage, we do not specify the explicit form of these operators - these can be, for example, quarkantiquark or two meson operators, etc.

In order to distinguish between valence and sea quarks, we use the standard trick (see, e.g., [44] and references therein), rewriting the above path integral in the following manner

$$
\begin{aligned}
C(t)= & \frac{1}{Z} \int \mathcal{D} U \mathcal{D} \psi_{\mathbf{v}} \mathcal{D} \bar{\psi}_{\mathbf{v}} \mathcal{D} \psi_{\mathbf{s}} \mathcal{D} \bar{\psi}_{\mathbf{s}} \mathcal{D} \psi_{\mathbf{g}} \mathcal{D} \bar{\psi}_{\mathrm{g}} \mathcal{O}_{\mathbf{v}}(t) \mathcal{O}_{\mathbf{v}}^{\dagger}(0) \\
& \times \exp \left\{-S_{G}-\int d^{4} x\left[\bar{\psi}_{\mathbf{v}}\left(\not D+m_{\mathrm{val}}\right) \psi_{\mathbf{v}}+\bar{\psi}_{\mathbf{s}}\left(\not D+m_{\mathrm{sea}}\right) \psi_{\mathbf{s}}+\bar{\psi}_{\mathbf{g}}\left(\not D+m_{\mathrm{gh}}\right) \psi_{\mathbf{g}}\right]\right\}
\end{aligned}
$$

Here, the subscripts "v," "s" and "g" stand for valence, sea and ghost quarks, the latter being described by commuting spinor fields. After performing the path integral over quarks, it is seen that the fermion determinant, coming from valence quarks, is exactly cancelled by the one from the ghost quarks, and the expression, given in eq. (2.1), is reproduced.

In order to describe the situation with partially twisted boundary conditions, one imposes twisted boundary conditions on the valence and ghost quarks and periodic boundary conditions on the sea quarks. The masses of all species of quarks are taken equal, in difference to the partially quenched case where $m=m_{\mathrm{val}}=m_{\mathrm{gh}} \neq m_{\text {sea }}$. Note that $m_{\mathrm{val}}, m_{\mathrm{gh}}, m_{\mathrm{sea}}$ are matrices in flavor space. Note also that we assume isospin symmetry throughout the paper $m_{u}=m_{d}=\hat{m} \neq m_{s}$. 
In the chiral limit, the infinite-volume theory is invariant under the graded symmetry group $\mathrm{SU}(2 \mathrm{~N} \mid \mathrm{N})_{\mathrm{L}} \times \mathrm{SU}(2 \mathrm{~N} \mid \mathrm{N})_{\mathrm{R}} \times \mathrm{U}(1)_{\mathrm{V}}$, where $N=3$ is the number of light flavors. The low-energy effective Lagrangian, ${ }^{1}$ corresponding to the case of partially twisted boundary conditions, contains the matrix $U=\exp \{i \sqrt{2} \Phi / F\}$ of the pseudo-Goldstone fields $\Phi$, which transforms under this group as

$$
U \rightarrow L U R^{\dagger}, \quad L, R \in \mathrm{SU}(2 \mathrm{~N} \mid \mathrm{N}) .
$$

The Hermitian matrix $\Phi$ has the following representation

$$
\Phi=\left(\begin{array}{lll}
M_{\mathrm{vv}} & M_{\mathrm{sv}}^{\dagger} & M_{\mathrm{gv}}^{\dagger} \\
M_{\mathrm{sv}} & M_{\mathrm{ss}} & M_{\mathrm{gs}}^{\dagger} \\
M_{\mathrm{gv}} & M_{\mathrm{gs}} & M_{\mathrm{gg}}
\end{array}\right) .
$$

Here, each of the entries is itself a $N \times N$ matrix in flavor space, containing meson fields built up from certain quark species (e.g., from valence quark and valence antiquark, from sea quark and ghost antiquark, and so on). The fields $M_{\mathrm{gv}}$ and $M_{\mathrm{gs}}$ are anti-commuting pseudoscalar fields (ghost mesons). Further, the matrix $\Phi$ obeys the condition [44]

$$
\operatorname{str} \Phi=\operatorname{tr}\left(M_{\mathrm{vv}}+M_{\mathrm{ss}}-M_{\mathrm{gg}}\right)=0,
$$

where "str" stands for the supertrace.

The effective chiral Lagrangian takes the form

$$
\mathcal{L}=\frac{F_{0}^{2}}{4} \operatorname{str}\left(\partial_{\mu} U \partial^{\mu} U^{\dagger}\right)-\frac{F_{0}^{2}}{4} \operatorname{str}\left(\chi U+U \chi^{\dagger}\right)+\text { higher-order terms }
$$

where $\chi=2 m B_{0}$ is proportional to the quark mass matrix.

In the infinite volume, the above theory is completely equivalent to ordinary Chiral Perturbation Theory (ChPT), since the masses of the quarks of all species are set equal. In a finite volume, the difference arises due to the different boundary conditions, set on the different meson fields. These boundary conditions are uniquely determined by the boundary conditions imposed on the constituents.

We do not intend to use the framework of the partially twisted ChPT to carry out explicit calculations. We need this framework only to facilitate the derivation of the Lüscher equation. To this end, let us consider large boxes with $L \gg M_{\pi}^{-1}$, where $M_{\pi}$ is the lightest mass in the system (the pion mass). The characteristic 3-momenta in such a box are much smaller than all masses - consequently, the system can be described by a non-relativistic EFT, whose low-energy couplings are consistently matched to the relativistic theory with the Lagrangian given in eq. (2.6) (for a detailed review of the non-relativistic theory in the infinite volume, we refer the reader, e.g., to the refs. [46, 47]; non-relativistic effective field theories in a finite volume are considered in refs. [41-43].). The two-body scattering

\footnotetext{
${ }^{1}$ We assume throughout this paper that the partially quenched theory is a theory with a well-defined Hamiltonian and spectrum, the presence of the negative-norm states being a sole artefact of the partial quenching. Recent investigations that can be found in the literature [45], support the above conjecture.
} 
$T$-matrix in the non-relativistic theory obeys the multi-channel Lippmann-Schwinger (LS) equation (for simplicity, we write down this equation in the CM frame)

$$
T_{\alpha \beta}\left(\mathbf{p}, \mathbf{q} ; P_{0}\right)=V_{\alpha \beta}(\mathbf{p}, \mathbf{q})+\sum_{\gamma} \int \frac{d^{d} \mathbf{k}}{(2 \pi)^{d}} \frac{V_{\alpha \gamma}(\mathbf{p}, \mathbf{q}) T_{\gamma \beta}\left(\mathbf{p}, \mathbf{q} ; P_{0}\right)}{2 w_{1}^{(\gamma)}(\mathbf{k}) 2 w_{2}^{(\gamma)}(\mathbf{k})\left(w_{1}^{(\gamma)}(\mathbf{k})+w_{2}^{(\gamma)}(\mathbf{k})-P_{0}-i 0\right)},
$$

where the sum runs over all two-body channels labeled by the index $\gamma$, and $w_{1}^{(\gamma)}(\mathbf{k}), w_{2}^{(\gamma)}(\mathbf{k})$ stand for the (relativistic) energies of the first and the second particle in this channel. The potentials $V_{\alpha \beta}(\mathbf{p}, \mathbf{q})$ encode the short-range dynamics, including inelastic many-body channels, which are closed at low energies. ${ }^{2}$ These potentials are constructed perturbatively and contain the couplings of the non-relativistic effective Lagrangian.

We use dimensional regularization throughout. In this regularization, the potentials $V_{\alpha \beta}(\mathbf{p}, \mathbf{q})$ coincide with the $K$-matrix elements. Expanding into the partial waves gives:

$$
V_{\alpha \beta}(\mathbf{p}, \mathbf{q})=4 \pi \sum_{l m} Y_{l m}(\hat{\mathbf{p}}) V_{\alpha \beta}^{l}(|\mathbf{p}|,|\mathbf{q}|) Y_{l m}^{*}(\hat{\mathbf{q}}),
$$

where $\hat{\mathbf{k}}$ denotes a unit vector in the direction of $\mathbf{k}$. It is easy to see that in the elastic region for the channel $\alpha$, on the energy shell where $|\mathbf{p}|=|\mathbf{q}|=q_{0}$,

$$
V_{\alpha \alpha}^{l}\left(q_{0}, q_{0}\right)=\frac{8 \pi P_{0}}{q_{0}} \tan \delta_{l}^{(\alpha)}\left(q_{0}\right), \quad P_{0}=w_{1}^{(\alpha)}\left(q_{0}\right)+w_{2}^{(\alpha)}\left(q_{0}\right),
$$

where $\delta_{l}^{(\alpha)}$ denotes the elastic scattering phase shift with angular momentum $l$. In the following, we shall neglect all partial waves except $l=0$. The inclusion of partial-wave mixing will be considered in the future.

When the relativistic theory, described by the Lagrangian given in eq. (2.6), is matched to the non-relativistic EFT, a complication arises, which stems from the mixing of the states containing neutral mesons. Namely, in the equation (2.7), the states $\alpha, \beta, \gamma$ correspond to the physical two-particle states. These are not always described by the meson fields which are present in the matrix $\Phi$. The reason for this is that not all the components of $\Phi$ are independent due to the condition $\operatorname{str} \Phi=0$.

In order to study the issue of mixing, let us again start with the relativistic theory described by the Lagrangian in eq. (2.6). We restrict ourselves first to order $p^{2}$, and retain only diagonal terms in the matrix $\Phi=\operatorname{diag}\left(\phi_{1}, \ldots, \phi_{9}\right)$ (the non-diagonal terms do not mix). The quadratic piece in the $O\left(p^{2}\right)$ Lagrangian takes the form

$$
\begin{aligned}
\mathcal{L}_{0}^{(2)}= & \frac{1}{2} \sum_{i=1}^{6}\left(\partial_{\mu} \phi_{i}\right)^{2}-\frac{1}{2} \sum_{i=7}^{9}\left(\partial_{\mu} \phi_{i}\right)^{2} \\
& -\frac{M^{2}}{2}\left(\phi_{1}^{2}+\phi_{2}^{2}+\phi_{4}^{2}+\phi_{5}^{2}-\phi_{7}^{2}-\phi_{8}^{2}\right)-\frac{M_{s}^{2}}{2}\left(\phi_{3}^{2}+\phi_{6}^{2}-\phi_{9}^{2}\right), \\
M^{2}= & 2 \hat{m} B_{0}, \quad M_{s}^{2}=2 m_{s} B_{0} .
\end{aligned}
$$

\footnotetext{
${ }^{2}$ There is a caveat in this argument. For example, there are multi-pion channels below $K \bar{K}$ channel. However, since the couplings to these channels are very weak, they can be safely ignored without changing the result. For more discussion on this issue, see ref. [9].
} 
Introducing the following linear combinations

$$
\begin{aligned}
& \phi_{1}=\frac{1}{\sqrt{2}} \omega_{1}-\frac{1}{\sqrt{6}} \omega_{2}-\frac{1}{2} \omega_{5}+\frac{1}{2} \omega_{8}, \\
& \phi_{2}=-\frac{1}{\sqrt{2}} \omega_{1}-\frac{1}{\sqrt{6}} \omega_{2}-\frac{1}{2} \omega_{5}+\frac{1}{2} \omega_{8}, \\
& \phi_{3}=\frac{\sqrt{6}}{3} \omega_{2}+\frac{1}{\sqrt{2}} \omega_{3}-\frac{1}{\sqrt{2}} \omega_{6}, \\
& \phi_{4}=-\frac{1}{\sqrt{6}} \omega_{2}+\frac{1}{\sqrt{2}} \omega_{4}+\frac{1}{2} \omega_{5}+\frac{1}{2} \omega_{8}, \\
& \phi_{5}=-\frac{1}{\sqrt{6}} \omega_{2}-\frac{1}{\sqrt{2}} \omega_{4}+\frac{1}{2} \omega_{5}+\frac{1}{2} \omega_{8}, \\
& \phi_{6}=\frac{\sqrt{6}}{3} \omega_{2}+\frac{1}{\sqrt{2}} \omega_{3}+\frac{1}{\sqrt{2}} \omega_{6}, \\
& \phi_{7}=-\frac{1}{\sqrt{6}} \omega_{2}+\frac{1}{\sqrt{2}} \omega_{7}+\omega_{8} \\
& \phi_{8}=-\frac{1}{\sqrt{6}} \omega_{2}-\frac{1}{\sqrt{2}} \omega_{7}+\omega_{8} \\
& \phi_{9}=\frac{\sqrt{6}}{3} \omega_{2}+\sqrt{2} \omega_{3},
\end{aligned}
$$

it is straightforward to check that the quadratic piece of the Lagrangian can be rewritten in terms of the fields $\omega_{1}, \ldots, \omega_{8}$ :

$$
\begin{aligned}
\mathcal{L}_{0}^{(2)}= & \frac{1}{2}\left\{\left(\partial_{\mu} \omega_{1}\right)^{2}+\left(\partial_{\mu} \omega_{2}\right)^{2}-\left(\partial_{\mu} \omega_{3}\right)^{2}+\left(\partial_{\mu} \omega_{4}\right)^{2}+\left(\partial_{\mu} \omega_{5}\right)^{2}+\left(\partial_{\mu} \omega_{6}\right)^{2}-\left(\partial_{\mu} \omega_{7}\right)^{2}-\left(\partial_{\mu} \omega_{8}\right)^{2}\right\} \\
& -\frac{M^{2}}{2}\left(\omega_{1}^{2}+\omega_{4}^{2}+\omega_{5}^{2}-\omega_{7}^{2}-\omega_{8}^{2}\right)+\frac{M_{s}^{2}}{2}\left(\omega_{3}^{2}-\omega_{6}^{2}\right)-\frac{M_{\eta}^{2}}{2} \omega_{2}^{2},
\end{aligned}
$$

where $M_{\eta}^{2}=\frac{2}{3} M_{s}^{2}+\frac{1}{3} M^{2}$. Note that the condition $\operatorname{str} \Phi=0$ is automatically fulfilled for the fields given by eq. (2.11). The eight fields $\omega_{1}, \ldots, \omega_{8}$ are unconstrained as opposed to the nine fields $\phi_{1}, \ldots, \phi_{9}$. The propagators for the physical particles can be read off from eq. (2.12). The fields $\omega_{3}, \omega_{7}, \omega_{8}$ are ghost fields (they enter the Lagrangian with a "wrong" sign). Note also that the transformation given in eq. (2.11) can be written in the compact form

$$
\Phi_{\mathrm{diag}}=\sum_{j=1}^{8} \omega_{j} T_{j},
$$

where in $\Phi_{\text {diag }}$ all components except those on the diagonal are set to zero, and the explicit form of the matrices $T_{j}$ is given in appendix $\mathrm{A}$.

The fields $\omega_{i}$ describe physical particles at $O\left(p^{2}\right)$, and matching to the non-relativistic theory is most easily performed in this basis. The symmetry relations between various matrix elements get, however, very complicated in this basis. In order to circumvent this 
problem, we have chosen to work in another basis

$\pi_{\mathrm{vv}}^{0}=\omega_{1}, \quad \pi_{\mathrm{ss}}^{0}=\omega_{4}, \quad \pi_{\mathrm{gg}}^{0}=\omega_{7}$,

$\eta_{\mathrm{vv}}=-\omega_{2}+\frac{1}{\sqrt{6}}\left(-\omega_{5}+\omega_{8}-\sqrt{2} \omega_{3}+\sqrt{2} \omega_{6}\right), \quad \eta_{\mathrm{vv}}^{\prime}=\frac{1}{\sqrt{6}}\left(-\sqrt{2} \omega_{5}+\sqrt{2} \omega_{8}+\omega_{3}-\omega_{6}\right)$,

$\eta_{\mathrm{ss}}=-\omega_{2}+\frac{1}{\sqrt{6}}\left(\omega_{5}+\omega_{8}-\sqrt{2} \omega_{3}-\sqrt{2} \omega_{6}\right), \quad \eta_{\mathrm{ss}}^{\prime}=\frac{1}{\sqrt{6}}\left(\sqrt{2} \omega_{5}+\sqrt{2} \omega_{8}+\omega_{3}+\omega_{6}\right)$,

$\eta_{\mathrm{gg}}=-\omega_{2}+\frac{1}{\sqrt{6}}\left(2 \omega_{8}-2 \sqrt{2} \omega_{3}\right), \quad \eta_{\mathrm{gg}}^{\prime}=\frac{1}{\sqrt{3}}\left(2 \omega_{8}+\sqrt{2} \omega_{3}\right)$.

Note also that the fields $\phi_{i}$ and $\pi^{0}, \eta, \eta^{\prime}$ are related by usual $\mathrm{SU}(3)$ relations:

$$
\begin{aligned}
& \phi_{1}=\frac{1}{\sqrt{2}} \pi_{\mathrm{vv}}^{0}+\frac{1}{\sqrt{6}} \eta_{\mathrm{vv}}+\frac{1}{\sqrt{3}} \eta_{\mathrm{vv}}^{\prime} \\
& \phi_{2}=-\frac{1}{\sqrt{2}} \pi_{\mathrm{vv}}^{0}+\frac{1}{\sqrt{6}} \eta_{\mathrm{vv}}+\frac{1}{\sqrt{3}} \eta_{\mathrm{vv}}^{\prime} \\
& \phi_{3}=-\frac{2}{\sqrt{6}} \eta_{\mathrm{vv}}+\frac{1}{\sqrt{3}} \eta_{\mathrm{vv}}^{\prime}, \\
& \phi_{4}=\frac{1}{\sqrt{2}} \pi_{\mathrm{ss}}^{0}+\frac{1}{\sqrt{6}} \eta_{\mathrm{ss}}+\frac{1}{\sqrt{3}} \eta_{\mathrm{ss}}^{\prime}, \\
& \phi_{5}=-\frac{1}{\sqrt{2}} \pi_{\mathrm{ss}}^{0}+\frac{1}{\sqrt{6}} \eta_{\mathrm{ss}}+\frac{1}{\sqrt{3}} \eta_{\mathrm{ss}}^{\prime}, \\
& \phi_{6}=-\frac{2}{\sqrt{6}} \eta_{\mathrm{ss}}+\frac{1}{\sqrt{3}} \eta_{\mathrm{ss}}^{\prime}, \\
& \phi_{7}=\frac{1}{\sqrt{2}} \pi_{\mathrm{gg}}^{0}+\frac{1}{\sqrt{6}} \eta_{\mathrm{gg}}+\frac{1}{\sqrt{3}} \eta_{\mathrm{gg}}^{\prime} \\
& \phi_{8}=-\frac{1}{\sqrt{2}} \pi_{\mathrm{gg}}^{0}+\frac{1}{\sqrt{6}} \eta_{\mathrm{gg}}+\frac{1}{\sqrt{3}} \eta_{\mathrm{gg}}^{\prime}, \\
& \phi_{9}=-\frac{2}{\sqrt{6}} \eta_{\mathrm{gg}}+\frac{1}{\sqrt{3}} \eta_{\mathrm{gg}}^{\prime},
\end{aligned}
$$

The propagator matrix is defined as

$$
i\left\langle 0\left|T \varphi_{A}(x) \varphi_{B}(0)\right| 0\right\rangle=\int \frac{d^{4} p}{(2 \pi)^{4}} e^{-i p x} D_{\varphi_{A} \varphi_{B}}(p), \quad A, B=\mathrm{vv}, \mathrm{ss}, \mathrm{gg},
$$

and $\varphi$ stands for $\pi^{0}, \eta$ or $\eta^{\prime}$. Further, due to isospin symmetry this matrix is diagonal in the subspace with different species of $\pi^{0}$ :

$$
D_{\pi_{\mathrm{vv}}^{0} \pi_{\mathrm{vv}}^{0}}(p)=D_{\pi_{\mathrm{ss}}^{0} \pi_{\mathrm{ss}}^{0}}(p)=-D_{\pi_{\mathrm{gg}}^{0} \pi_{\mathrm{gg}}^{0}}(p)=D_{\pi}
$$

However, different species of the $\eta$ and $\eta^{\prime}$ mix. Defining the $2 \times 2$ matrix

$$
\Omega_{A B}(p)=\left(\begin{array}{cc}
D_{\eta_{A} \eta_{B}}(p) & D_{\eta_{A} \eta_{B}^{\prime}}(p) \\
D_{\eta_{A}^{\prime} \eta_{B}}(p) & D_{\eta_{A}^{\prime} \eta_{B}^{\prime}}(p)
\end{array}\right)
$$

we get

$$
\begin{aligned}
& \Omega_{\mathrm{vv}, \mathrm{vv}}=\Omega_{\mathrm{ss}, \mathrm{ss}}=A, \quad \Omega_{\mathrm{gg}, \mathrm{gg}}=A+2 X, \\
& \Omega_{\mathrm{vv}, \mathrm{ss}}=\Omega_{\mathrm{vv}, \mathrm{gg}}=\Omega_{\mathrm{ss}, \mathrm{vv}}=\Omega_{\mathrm{ss}, \mathrm{gg}}=\Omega_{\mathrm{gg}, \mathrm{vv}}=\Omega_{\mathrm{gg}, \mathrm{ss}}=A+X,
\end{aligned}
$$


where

$$
A=\left(\begin{array}{cc}
D_{\eta} & 0 \\
0 & 0
\end{array}\right), \quad X=\left(\begin{array}{cc}
-\frac{1}{3} D_{\pi}-\frac{2}{3} D_{s} & -\frac{\sqrt{2}}{3}\left(D_{\pi}-D_{s}\right) \\
-\frac{\sqrt{2}}{3}\left(D_{\pi}-D_{s}\right) & -\frac{2}{3} D_{\pi}-\frac{1}{3} D_{s}
\end{array}\right) .
$$

In the above equations, the following notations were used:

$$
D_{\pi}=\frac{1}{M^{2}-p^{2}}, \quad D_{\eta}=\frac{1}{M_{\eta}^{2}-p^{2}}, \quad D_{s}=\frac{1}{M_{s}^{2}-p^{2}} .
$$

If matching to the non-relativistic theory is performed in this basis, the free two-particle Green function is no more diagonal in the channel basis and the equation (2.7) is replaced by ${ }^{3}$

$$
T_{i j}\left(\mathbf{p}, \mathbf{q} ; P_{0}\right)=V_{i j}(\mathbf{p}, \mathbf{q})+\sum_{n m} \int \frac{d^{d} \mathbf{k}}{(2 \pi)^{d}} V_{i n}(\mathbf{p}, \mathbf{q}) \tilde{G}_{n m}\left(\mathbf{k} ; P_{0}\right) T_{m j}\left(\mathbf{p}, \mathbf{q} ; P_{0}\right) .
$$

The entries of the matrix $\tilde{G}_{n m}$ can be easily determined by using eqs. (2.17), (2.18), (2.19) and (2.20), see below. As already mentioned, the advantage of such a choice of the basis is that the symmetry relations for the matrix elements $V_{i j}, T_{i j}$ are less complicated in this basis.

An important remark is in order. Up to now, we have considered the mixing of the neutral states only at $O\left(p^{2}\right)$ in ChPT. The coefficients, e.g., in eq. (2.11) will change, if higher-order terms are included. How will this affect our expressions? In order not to obscure the crucial physical arguments, we shall neglect higher-order corrections for now. At the end, we return to this question and show that the final result remains unaffected by these corrections.

\section{Symmetries of the potential}

As already mentioned in the introduction, we concentrate on S-wave scattering in the sector with total isospin $I=1$. It is convenient to choose $I_{3}=1$. Tracking the quarks of different species, flowing through the diagrams describing meson-meson scattering, and starting from the state that contains only valence quarks, it is easy to see that the LS equation couples 11 different channels, as given in table 1 .

As immediately seen from this table, the valence sector couples to the sea and ghost sectors through the annihilation diagrams of the type shown in figure 1. In addition, $\pi^{+} \pi^{0}$ states with quarks of different species are no more forbidden in the S-wave. ${ }^{4}$ Hence, in general, the partially twisted Lüscher equation will differ from the fully twisted one.

\footnotetext{
${ }^{3}$ We shall use Greek indices $\alpha, \beta, \gamma, \ldots$, to label channels in the basis where the matrix of the two-point functions of the meson fields is diagonal. This corresponds to working with the fields $\omega_{1}, \cdots, \omega_{8}$. On the other hand, in the transformed basis (see eq. (2.14)), we label the channels by Latin letters $i, j, n, m, \ldots$. .

${ }^{4}$ As it is easily seen, the components $\left|\left(u_{v} \bar{d}_{s}\right)\left(u_{s} \bar{u}_{v}\right)\right\rangle$ and $\left|\left(d_{v} \bar{d}_{s}\right)\left(u_{s} \bar{d}_{v}\right)\right\rangle$ (similarly, with $s \rightarrow g$ ) in the entries 10 and 11 of the table 1 do not indeed contribute to the matrix elements under consideration. We have nevertheless retained them in order to preserve a (formal) analogy with the wave function of the $\pi^{0}$ in the diagonal sectors. Nothing changes in the results, if these components are omitted from the beginning.
} 


\begin{tabular}{|l|l|l|}
\hline Index & Channel & Quark content \\
\hline 1 & $\left|\pi_{\mathrm{vv}}^{+} \eta_{\mathrm{vv}}\right\rangle$ & $-\frac{1}{\sqrt{6}}\left|\left(u_{\mathrm{v}} \bar{d}_{\mathrm{v}}\right)\left(u_{\mathrm{v}} \bar{u}_{\mathrm{v}}+d_{\mathrm{v}} \bar{d}_{\mathrm{v}}-2 s_{\mathrm{v}} \bar{s}_{\mathrm{v}}\right)\right\rangle$ \\
2 & $\left|\pi_{\mathrm{vv}}^{+} \eta_{\mathrm{vv}}^{\prime}\right\rangle$ & $-\frac{1}{\sqrt{3}}\left|\left(u_{\mathrm{v}} \bar{d}_{\mathrm{v}}\right)\left(u_{\mathrm{v}} \bar{u}_{\mathrm{v}}+d_{\mathrm{v}} \bar{d}_{\mathrm{v}}+s_{\mathrm{v}} \bar{s}_{\mathrm{v}}\right)\right\rangle$ \\
3 & $\left|\pi_{\mathrm{vv}}^{+} \eta_{\mathrm{ss}}\right\rangle$ & $-\frac{1}{\sqrt{6}}\left|\left(u_{\mathrm{v}} \bar{d}_{\mathrm{v}}\right)\left(u_{\mathrm{s}} \bar{u}_{\mathrm{s}}+d_{\mathrm{s}} \bar{d}_{\mathrm{s}}-2 s_{\mathrm{s}} \bar{s}_{\mathrm{s}}\right)\right\rangle$ \\
4 & $\left|\pi_{\mathrm{vv}}^{+} \eta_{\mathrm{ss}}^{\prime}\right\rangle$ & $-\frac{1}{\sqrt{3}}\left|\left(u_{\mathrm{v}} \bar{d}_{\mathrm{v}}\right)\left(u_{\mathrm{s}} \bar{u}_{\mathrm{s}}+d_{\mathrm{s}} \bar{d}_{\mathrm{s}}+s_{\mathrm{s}} \bar{s}_{\mathrm{s}}\right)\right\rangle$ \\
5 & $\left|\pi_{\mathrm{vv}}^{+} \eta_{\mathrm{gg}}\right\rangle$ & $-\frac{1}{\sqrt{6}}\left|\left(u_{\mathrm{v}} \bar{d}_{\mathrm{v}}\right)\left(u_{\mathrm{g}} \bar{u}_{\mathrm{g}}+d_{\mathrm{g}} \bar{d}_{\mathrm{g}}-2 s_{\mathrm{g}} \bar{s}_{\mathrm{g}}\right)\right\rangle$ \\
6 & $\left|\pi_{\mathrm{vv}}^{+} \eta_{\mathrm{gg}}^{\prime}\right\rangle$ & $-\frac{1}{\sqrt{3}}\left|\left(u_{\mathrm{v}} \bar{d}_{\mathrm{v}}\right)\left(u_{\mathrm{g}} \bar{u}_{\mathrm{g}}+d_{\mathrm{g}} \bar{d}_{\mathrm{g}}+s_{\mathrm{g}} \bar{s}_{\mathrm{g}}\right)\right\rangle$ \\
7 & $\left|K_{\mathrm{vv}}^{+} \bar{K}_{\mathrm{vv}}^{0}\right\rangle$ & $\left|\left(u_{\mathrm{v}} \bar{s}_{\mathrm{v}}\right)\left(s_{\mathrm{v}} \bar{d}_{\mathrm{v}}\right)\right\rangle$ \\
8 & $\left|K_{\mathrm{vs}}^{+} \bar{K}_{\mathrm{sv}}^{0}\right\rangle$ & $\left|\left(u_{\mathrm{v}} \bar{s}_{\mathrm{s}}\right)\left(s_{\mathrm{s}} \bar{d}_{\mathrm{v}}\right)\right\rangle$ \\
9 & $\left|K_{\mathrm{vg}}^{+} \bar{K}_{\mathrm{gv}}^{0}\right\rangle$ & $\left|\left(u_{\mathrm{v}} \bar{s}_{\mathrm{g}}\right)\left(s_{\mathrm{g}} \bar{d}_{\mathrm{v}}\right)\right\rangle$ \\
10 & $\left|\pi_{\mathrm{vs}}^{+} \pi_{\mathrm{sv}}^{0}\right\rangle$ & $\frac{1}{2}\left(-\left(u_{\mathrm{v}} \bar{d}_{\mathrm{s}}\right)\left(u_{\mathrm{s}} \bar{u}_{\mathrm{v}}-d_{\mathrm{s}} \bar{d}_{\mathrm{v}}\right)+\left(u_{\mathrm{v}} \bar{u}_{\mathrm{s}}-d_{\mathrm{v}} \bar{d}_{\mathrm{s}}\right)\left(u_{\mathrm{s}} \bar{d}_{\mathrm{v}}\right)\right\rangle$ \\
11 & $\left|\pi_{\mathrm{vg}}^{+} \pi_{\mathrm{gv}}^{0}\right\rangle$ & $\frac{1}{2}\left(-\left(u_{\mathrm{v}} \bar{d}_{\mathrm{g}}\right)\left(u_{\mathrm{g}} \bar{u}_{\mathrm{v}}-d_{\mathrm{g}} \bar{d}_{\mathrm{v}}\right)+\left(u_{\mathrm{v}} \bar{u}_{\mathrm{g}}-d_{\mathrm{v}} \bar{d}_{\mathrm{g}}\right)\left(u_{\mathrm{g}} \bar{d}_{\mathrm{v}}\right)\right\rangle$ \\
\hline
\end{tabular}

Table 1. Scattering channels for the case of $I=I_{3}=1$.

For comparison, let us consider a (trivial) example of meson scattering in the channel with $I=2$, where the answer is already known. Take, for simplicity, $I_{3}=2$. In this case, starting in the valence quark sector, one gets only one state $\left|\pi_{\mathrm{vv}} \pi_{\mathrm{vv}}^{+}\right\rangle=\left|\left(u_{\mathrm{v}} \bar{d}_{\mathrm{v}}\right)\left(u_{\mathrm{v}} \bar{d}_{\mathrm{v}}\right)\right\rangle$. Annihilation diagrams are absent and, consequently, partial and full twisting are equivalent up to exponentially suppressed contributions.

Using dimensional regularization, it is easy to see that the LS equation reduces to an algebraic matrix equation (see, e.g., refs. [42, 43])

$$
T_{i j}=V_{i j}+\sum_{n m} V_{i m} G_{n m} T_{m j}
$$

This equation relates the on-shell matrix elements of $T$ and $V$. 
The free Green function $G_{n m}$ in the channel with $I=1$ is given by the $11 \times 11$ matrix

$$
G=\left(\begin{array}{cccccccc}
B & B+Y & B+Y & O_{1} & O_{1} & O_{1} & O_{1} & O_{1} \\
B+Y & B & B+Y & O_{1} & O_{1} & O_{1} & O_{1} & O_{1} \\
B+Y & B+Y & B+2 Y & O_{1} & O_{1} & O_{1} & O_{1} & O_{1} \\
O_{1}^{T} & O_{1}^{T} & O_{1}^{T} & K & 0 & 0 & 0 & 0 \\
O_{1}^{T} & O_{1}^{T} & O_{1}^{T} & 0 & K & 0 & 0 & 0 \\
O_{1}^{T} & O_{1}^{T} & O_{1}^{T} & 0 & 0 & -K & 0 & 0 \\
O_{1}^{T} & O_{1}^{T} & O_{1}^{T} & 0 & 0 & 0 & P & 0 \\
O_{1}^{T} & O_{1}^{T} & O_{1}^{T} & 0 & 0 & 0 & 0 & -P
\end{array}\right),
$$

where $B$ and $Y$ are $2 \times 2$ matrices (cf. with eq. (2.20)), and $O_{1}$ is a $2 \times 1$ matrix:

$$
O_{1}=\left(\begin{array}{l}
0 \\
0
\end{array}\right), \quad B=\left(\begin{array}{cc}
E & 0 \\
0 & 0
\end{array}\right), \quad Y=\left(\begin{array}{cc}
-\frac{1}{3} P-\frac{2}{3} S-\frac{\sqrt{2}}{3}(P-S) \\
-\frac{\sqrt{2}}{3}(P-S)-\frac{2}{3} P-\frac{1}{3} S
\end{array}\right),
$$

and the quantities $K, E, P, S$ are loops with free Green functions in the non-relativistic EFT, corresponding to the different two-particle channels:

$$
\begin{aligned}
K \bar{K}: & K=\int \frac{d^{d} k}{(2 \pi)^{d}} \frac{1}{\left(2 w_{K}(\mathbf{k})\right)^{2}} \frac{1}{2 w_{K}(\mathbf{k})-P_{0}} \\
\pi \eta: & E=\int \frac{d^{d} k}{(2 \pi)^{d}} \frac{1}{2 w_{\pi}(\mathbf{k}) 2 w_{\eta}(\mathbf{k})} \frac{1}{w_{\pi}(\mathbf{k})+w_{\eta}(\mathbf{k})-P_{0}} \\
\pi \pi: & P=\int \frac{d^{d} k}{(2 \pi)^{d}} \frac{1}{\left(2 w_{\pi}(\mathbf{k})\right)^{2}} \frac{1}{2 w_{\pi}(\mathbf{k})-P_{0}}, \\
\pi \eta^{s}: & S=\int \frac{d^{d} k}{(2 \pi)^{d}} \frac{1}{2 w_{\pi}(\mathbf{k}) 2 w_{s}(\mathbf{k})} \frac{1}{w_{\pi}(\mathbf{k})+w_{s}(\mathbf{k})-P_{0}}
\end{aligned}
$$

Here, $w_{s}(\mathbf{k})=\left(M_{s}^{2}+\mathbf{k}^{2}\right)^{1 / 2}$, where $M_{s}$ denotes the physical mass of the $\eta^{s} \doteq \bar{s} s$ meson, which emerges in the partially twisted ChPT (to the lowest order, $M_{s}^{2}=2 m_{s} B_{0}$, see eq. (2.10)).

Calculating the above integrals by using the technique, described in ref. [47], we finally get

$$
K, E, P, S=\frac{i p}{8 \pi P_{0}}, \quad p=\frac{\lambda^{1 / 2}\left(P_{0}^{2}, m_{1}^{2}, m_{2}^{2}\right)}{2 P_{0}} .
$$

Here, $p$ stands for the relative momentum of the pair of particles in the intermediate state, $m_{1}, m_{2}$ are masses of these particles, and $\lambda(x, y, z)=x^{2}+y^{2}+z^{2}-2 x y-2 y z-2 z x$ denotes the triangle function. 
The potential $V$ and the $T$-matrix are also $11 \times 11$ matrices. The $T$-matrix can be written in the following form

$$
T=\left(\begin{array}{ccccccccccc}
c & d & \omega & \omega^{\prime} & -\omega & -\omega^{\prime} & b & y^{\prime} & y^{\prime} & y^{\prime \prime} & y^{\prime \prime} \\
d & c^{\prime} & \nu & \nu^{\prime} & -\nu & -\nu^{\prime} & b^{\prime} & z^{\prime} & z^{\prime} & z^{\prime \prime} & z^{\prime \prime} \\
\omega & \nu & f & f^{\prime} & f^{\prime \prime} & -\hat{f} & -\lambda & t & t^{\prime} & u & u^{\prime} \\
\omega^{\prime} & \nu^{\prime} & f^{\prime} & f_{0} & -\hat{f} & f^{\prime \prime \prime} & -\lambda^{\prime} & h & h^{\prime} & r & r^{\prime} \\
-\omega & -\nu & f^{\prime \prime} & -\hat{f} & \tilde{f} & \tilde{f}^{\prime} & \lambda & -t^{\prime} & -\tilde{t} & -u^{\prime} & -\tilde{u} \\
-\omega^{\prime} & -\nu^{\prime} & -\hat{f} & f^{\prime \prime \prime} & \tilde{f}^{\prime} & \tilde{f}_{0} & \lambda^{\prime} & -h^{\prime} & -\tilde{h} & -r^{\prime} & -\tilde{r} \\
b & b^{\prime} & -\lambda & -\lambda^{\prime} & \lambda & \lambda^{\prime} & a & y & y & z & z \\
y^{\prime} & z^{\prime} & t & h & -t^{\prime} & -h^{\prime} & y & a & y & z & z \\
y^{\prime} & z^{\prime} & t^{\prime} & h^{\prime} & -\tilde{t} & -\tilde{h} & y & y & \tilde{a} & z & z \\
y^{\prime \prime} & z^{\prime \prime} & u & r & -u^{\prime} & -r^{\prime} & z & z & z & q & q^{\prime} \\
y^{\prime \prime} & z^{\prime \prime} & u^{\prime} & r^{\prime} & -\tilde{u} & -\tilde{r} & z & z & z & q^{\prime} & \tilde{q}
\end{array}\right) .
$$

Here, $c, d, \omega, \ldots$ denote the entries of the matrix $T_{i j}$. Some (trivial) symmetry relations are already taken into account, for example, $T_{36}=T_{45}=-\hat{f}$. Note also that the matrix $T_{i j}$ is symmetric. On the mass shell, the entries of the above matrix are the functions of a single Mandelstam variable $s$ (we remind the reader that all partial waves except the S-wave are neglected). We use the name physical for the amplitudes that describe the scattering in the sector of only valence quarks: $T_{77}=a$ corresponds to the $K \bar{K}$ elastic scattering, $T_{11}=c$ to the $\pi \eta$ elastic scattering and $T_{17}=T_{71}=b$ to the $K \bar{K} \rightarrow \pi \eta$ transition amplitude. Other entries in this matrix are "unphysical." For example, $y$ corresponds to the transition between the valence and sea quark sectors. Considering the quark diagrams for this process (see figure 2 and eq. (3.7) below), one straightforwardly ensures that $y$ corresponds to the contribution of the disconnected diagrams to the $K \bar{K}$ elastic amplitude.

There exist more symmetry relations, which relate various entries in the above matrix. A straightforward way to derive these relations in general is to express these amplitudes in terms of the quark propagators and take into account the fact that the valence, sea and ghost quark masses all coincide. Below, we give few examples of such calculations. ${ }^{5}$

\footnotetext{
${ }^{5}$ A crucial property of the Lippmann-Schwinger equation with the Green function given in eq. (3.2) is that the symmetries of the matrix $T$ are the same as the symmetries of the potential matrix $V$. Later, we shall check this property explicitly.
} 

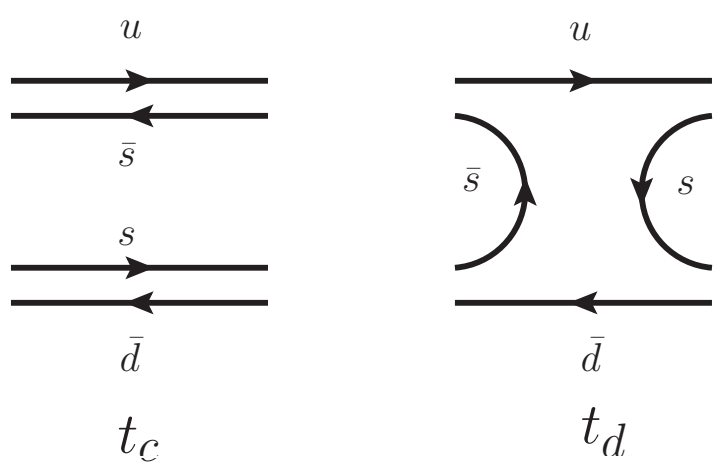

Figure 2. Connected $\left(t_{c}\right)$ and disconnected $\left(t_{d}\right)$ diagrams, emerging in $K \bar{K} \rightarrow K \bar{K}$ scattering amplitudes with various quark species, see eq. (3.7).

Example 1. Consider the quark diagrams for the transition between various $K \bar{K}$ states. The full 4-point Green functions of the bilinear quark operators are given by

$$
\begin{aligned}
& \Gamma_{77}=t_{c}-t_{d}, \\
& \Gamma_{88}=t_{c}-t_{d}, \\
& \Gamma_{99}=-t_{c}-t_{d}, \\
& \Gamma_{78}=\Gamma_{79}=\Gamma_{89}=\Gamma_{87}=\Gamma_{97}=\Gamma_{98}=-t_{d},
\end{aligned}
$$

where $t_{c}$ and $t_{d}$ denote connected and disconnected diagrams, respectively, as shown in figure 2. Different signs in different matrix elements emerge from calculations with anticommuting (valence, sea) and commuting (ghost) fields. The connected diagrams are, of course, absent in the non-diagonal matrix elements. Note that the quark propagators, used in the diagrams, are the same for all quark species, since that masses of valence, sea and ghost quarks are the same.

The scattering matrix elements are given by the residues of the 4-point Green functions at the poles, corresponding to the external mesonic legs. It is seen that all Green functions in eq. (3.7) are expressed only through two quantities and, hence, there are some linear relations between them. It can be shown (see section 5 for the details) that the scattering matrix elements obey exactly the same linear relations even if $\hat{m} \neq m_{s}$. Introducing the notations $T_{77}=a$, and $T_{78}=y$, we finally arrive at the relations

$$
T_{88}=a, \quad T_{99}=\tilde{a}=-a+2 y, \quad T_{78}=T_{79}=T_{89}=T_{87}=T_{97}=T_{98}=y .
$$

Example 2. Consider

$$
\Gamma_{33}=\frac{1}{6}\left\{\left[4 W_{l l}-8 W_{l s}+4 W_{s s}\right]+2 x_{l}+4 x_{s}\right\},
$$

where the terms in square brackets stem from the tadpole diagrams, see figure 3 , and the subscripts "l" and "s" stand for "light" and "strange." 

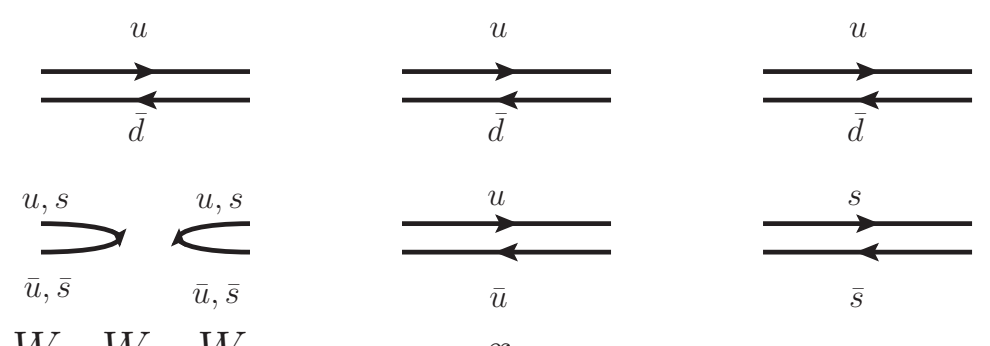

$W_{l l}, W_{l s}, W_{s s}$
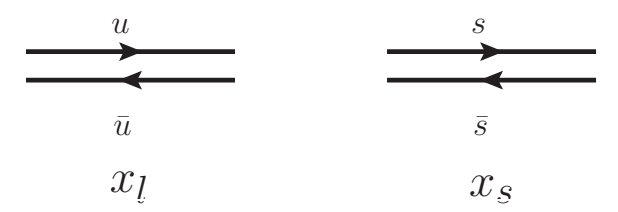

Figure 3. Diagrams contributing to $\pi \eta$ scattering in the valence quark sector, see eq. (3.9). $W_{l l}, W_{l s}, W_{s s}$ correspond to the diagrams with zero, one, two strange quarks in the tadpoles. $x_{l}$ and $x_{s}$ are connected diagrams without and with strange quarks.

Carrying out similar calculations, we get

$$
\begin{aligned}
& \Gamma_{34}=\frac{1}{3 \sqrt{2}}\left\{\left[4 W_{l l}-2 W_{l s}+2 W_{s s}\right]+2 x_{l}-2 x_{s}\right\}, \\
& \Gamma_{35}=-\frac{1}{6}\left\{\left[4 W_{l l}-8 W_{l s}+4 W_{s s}\right]\right\}, \\
& \Gamma_{45}=-\frac{1}{3 \sqrt{2}}\left\{\left[4 W_{l l}-2 W_{l s}+2 W_{s s}\right]\right\}, \\
& \Gamma_{44}=\frac{1}{3}\left\{\left[4 W_{l l}+4 W_{l s}+W_{s s}\right]+2 x_{l}+x_{s}\right\}, \\
& \Gamma_{46}=-\frac{1}{3}\left\{\left[4 W_{l l}+4 W_{l s}+W_{s s}\right]\right\}, \\
& \Gamma_{55}=\frac{1}{6}\left\{\left[4 W_{l l}-8 W_{l s}+4 W_{s s}\right]-2 x_{l}-4 x_{s}\right\}, \\
& \Gamma_{56}=\frac{1}{3 \sqrt{2}}\left\{\left[4 W_{l l}-2 W_{l s}+2 W_{s s}\right]-2 x_{l}+2 x_{s}\right\}, \\
& \Gamma_{66}=\frac{1}{3}\left\{\left[4 W_{l l}+4 W_{l s}+W_{s s}\right]-2 x_{l}-x_{s}\right\} .
\end{aligned}
$$

From these relations one easily gets

$$
\begin{aligned}
f+\tilde{f} & =-2 f^{\prime \prime}, \\
f^{\prime}+\tilde{f}^{\prime} & =2 \hat{f} \\
f_{0}+\tilde{f}_{0} & =-2 f^{\prime \prime \prime}, \\
f^{\prime}-\tilde{f}^{\prime} & =-\sqrt{2}\left(f-\tilde{f}-f_{0}+\tilde{f}_{0}\right) .
\end{aligned}
$$

Acting in the same manner as described in the examples, we get more relations. The 
ones listed below will be used further:

$$
\begin{aligned}
t+\tilde{t} & =2 t^{\prime}, \\
y^{\prime}+t-t^{\prime} & =y^{\prime}+t^{\prime}-\tilde{t}=b \\
h+\tilde{h} & =2 h^{\prime}, \\
z^{\prime}+h-h^{\prime} & =z^{\prime}+h^{\prime}-\tilde{h}=b^{\prime} \\
u+\tilde{u} & =2 u^{\prime}, \\
r+\tilde{r} & =2 r^{\prime}, \\
\sqrt{2}(\tilde{u}-u) & =\tilde{r}-r, \\
-\sqrt{2}(\tilde{h}-h) & =\tilde{t}-t, \\
\lambda & =-t^{\prime}, \\
\lambda^{\prime} & =-h^{\prime}, \\
z^{\prime}-\sqrt{2} y^{\prime} & =h^{\prime}-\sqrt{2} t^{\prime}, \\
\sqrt{2} d & =2 c-2 \omega+\sqrt{2} \omega^{\prime}+\sqrt{2}\left(f^{\prime}-\tilde{f}^{\prime}\right)-\left(f_{0}-\tilde{f}_{0}\right), \\
q+\tilde{q} & =2 q^{\prime}, \\
\nu & =\sqrt{2} \omega+\hat{f}+\sqrt{2} f^{\prime \prime}, \\
\nu^{\prime} & =\sqrt{2} \omega^{\prime}-\sqrt{2} \hat{f}-f^{\prime \prime \prime}, \\
c^{\prime} & =c+\frac{d}{\sqrt{2}}-f^{\prime \prime \prime}-2 f^{\prime \prime}-2 \sqrt{2} \hat{f}-3 \omega+\frac{3}{\sqrt{2}} \omega^{\prime} \\
\sqrt{2} b^{\prime} & =\sqrt{2} z^{\prime}+y^{\prime}-b, \\
\sqrt{2} y^{\prime \prime}-z^{\prime \prime} & =\sqrt{2} u-r .
\end{aligned}
$$

As the next step, we would like to establish, what are the implications of the above symmetry relations for the potential matrix $V_{i j}$. Recalling that in dimensional regularization the $T$-matrix obeys the algebraic LS equation (3.1), where $T$ and $G$ are given by $11 \times 11$ matrices in eqs. (3.6) and (3.2), respectively, it is a straightforward task to solve the above matrix equation with respect to $V$. In doing so, we find it useful to first perform the linear 
transformation of the LS equation with the matrix

$$
O=\left(\begin{array}{ccccccccccc}
1 / 2 & 0 & 1 / 2 & 0 & 0 & 0 & 0 & 0 & 0 & 0 & 0 \\
0 & 1 / 2 & 0 & 1 / 2 & 0 & 0 & 0 & 0 & 0 & 0 & 0 \\
1 / 4 & 0 & -1 / 4 & 0 & 1 / 2 & 0 & 0 & 0 & 0 & 0 & 0 \\
0 & 1 / 4 & 0 & -1 / 4 & 0 & 1 / 2 & 0 & 0 & 0 & 0 & 0 \\
1 / 4 & 0 & -1 / 4 & 0 & -1 / 2 & 0 & 0 & 0 & 0 & 0 & 0 \\
0 & 1 / 4 & 0 & -1 / 4 & 0 & -1 / 2 & 0 & 0 & 0 & 0 & 0 \\
0 & 0 & 0 & 0 & 0 & 0 & 1 & 0 & 0 & 0 & 0 \\
0 & 0 & 0 & 0 & 0 & 0 & 0 & 1 & 0 & 0 & 0 \\
0 & 0 & 0 & 0 & 0 & 0 & 0 & 0 & 1 & 0 & 0 \\
0 & 0 & 0 & 0 & 0 & 0 & 0 & 0 & 0 & 1 & 0 \\
0 & 0 & 0 & 0 & 0 & 0 & 0 & 0 & 0 & 0 & 1
\end{array}\right) .
$$

The transformed Green function is given by the matrix

$$
\hat{G}=O^{T} G O=\left(\begin{array}{cccccccc}
B+\frac{3}{4} Y & -\frac{1}{4} Y & -\frac{1}{4} Y & O_{1} & O_{1} & O_{1} & O_{1} & O_{1} \\
-\frac{1}{4} Y & -\frac{1}{4} Y & \frac{1}{4} Y & O_{1} & O_{1} & O_{1} & O_{1} & O_{1} \\
-\frac{1}{4} Y & \frac{1}{4} Y & O_{2} & O_{1} & O_{1} & O_{1} & O_{1} & O_{1} \\
O_{1}^{T} & O_{1}^{T} & O_{1}^{T} & K & 0 & 0 & 0 & 0 \\
O_{1}^{T} & O_{1}^{T} & O_{1}^{T} & 0 & K & 0 & 0 & 0 \\
O_{1}^{T} & O_{1}^{T} & O_{1}^{T} & 0 & 0 & -K & 0 & 0 \\
O_{1}^{T} & O_{1}^{T} & O_{1}^{T} & 0 & 0 & 0 & P & 0 \\
O_{1}^{T} & O_{1}^{T} & O_{1}^{T} & 0 & 0 & 0 & 0 & -P
\end{array}\right),
$$

where

$$
O_{2}=\left(\begin{array}{ll}
0 & 0 \\
0 & 0
\end{array}\right) .
$$


Note that this linear transformation minimizes the number of the entries in the free Green function, corresponding to the physical $\pi \eta$ state. Namely, as seen from eq. (3.14), the $\pi \eta$ state appears only once, on the main diagonal of the matrix $\hat{G}$. The physical $K \bar{K}$ intermediate states also appear only on the diagonal. Consequently, after using Feshbach's method $[48,49]$ to define the effective potential that includes all unphysical intermediate states, the resulting LS equation with this effective potential is expected to have a particularly simple form. We shall explicitly see this below.

The transformed LS equation (3.1) takes the form

$$
\hat{T}=\hat{V}+\hat{V} \hat{G} \hat{T}, \quad \hat{T}=O^{-1} T\left(O^{T}\right)^{-1}, \quad \hat{V}=O^{-1} V\left(O^{T}\right)^{-1} .
$$

For the analysis of the symmetries of the potential $V$, it is convenient to further split the Green function

$$
\hat{G}=\hat{G}_{0}+\hat{G}_{1}, \quad \hat{G}_{0}=\operatorname{diag}(E, 0,0,0,0,0, K, K,-K, 0,0) .
$$

The split LS equation is:

$$
\hat{T}=\hat{W}+\hat{W} \hat{G}_{0} \hat{T}, \quad \hat{W}=\hat{V}+\hat{V} \hat{G}_{1} \hat{W} .
$$

A crucial point is that the certain matrix elements of the matrix $W$ do not contain $\pi \pi$ and $\pi \eta^{s}$ loops.

$$
\hat{V}_{11}=\hat{W}_{11}, \quad \hat{V}_{17}=\hat{W}_{17}, \quad \hat{V}_{18}=\hat{W}_{18}, \quad \hat{V}_{19}=\hat{W}_{19}, \quad \hat{V}_{77}=\hat{W}_{77}, \quad \hat{V}_{88}=\hat{W}_{88} .
$$

This property does not hold in general. For example,

$$
\hat{W}_{78}=\hat{W}_{79}=\hat{W}_{89}=\frac{1}{2}\left(\hat{W}_{99}+\hat{V}_{77}\right) \neq \hat{V}_{78} .
$$

The above property has direct implications for the matrix elements of $T$ as well. Namely, iterating the matrix $W$, it can be shown that in the physical matrix elements $a, b, c$ no $\pi \pi$ and $\pi \eta^{s}$ loops are present, whereas the unphysical matrix elements (e.g., $y$ ), in general, contain such loops. The above statements can be verified explicitly by direct calculations, in which the use of eqs. (3.8), (3.11) and (3.12) is crucial. ${ }^{6}$

Taking into account the above relations, it is now straightforwardly seen that the physical matrix elements $a, b, c$ are determined from a much simpler LS equation

$$
\tau=\sigma+\sigma g \tau
$$

where $\tau, g, \sigma$ are $4 \times 4$ matrices that are obtained from the matrices $\hat{T}, \hat{G}_{0}, \hat{W}$, respectively, by deleting all rows/columns with the indices $i, j=2,3,4,5,6,10,11$ (for these values of

\footnotetext{
${ }^{6}$ Inverting $11 \times 11$ matrices analytically have turned to be a very demanding task, leading to extremely lengthy expressions. What we have explicitly checked in analytic calculations is that the above statements are valid for first few terms in the Born expansion of the LS equation. In addition, taking random numerical input for the $T$-matrix elements, we have checked that all symmetry relations hold numerically for the matrix elements of the potential as well.
} 
the indices the matrix $\hat{G}_{0}$ has vanishing entries on the diagonal). Namely, these matrices are given by

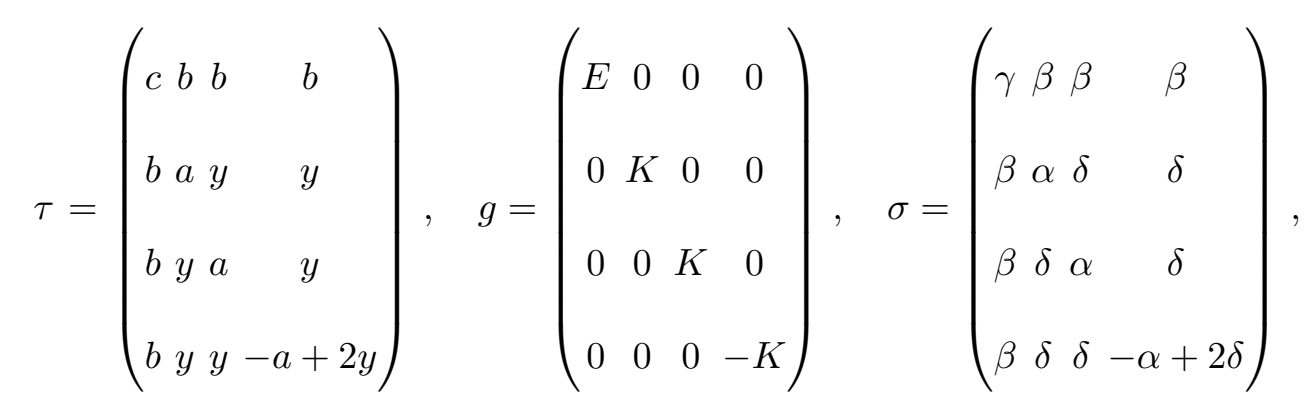

where

$$
\alpha=\hat{V}_{77}, \quad \beta=\hat{V}_{17}, \quad \gamma=\hat{V}_{11}, \quad \delta=\hat{W}_{78}
$$

The solution of the LS equation for the physical matrix elements gives:

$$
\begin{aligned}
& a=\frac{\alpha-E\left(\alpha \gamma-\beta^{2}\right)}{D}, \quad b=\frac{\beta}{D}, \quad c=\frac{\gamma-K\left(\alpha \gamma-\beta^{2}\right)}{D}, \\
& D=(1-K \alpha)(1-E \gamma)-K E \beta^{2} .
\end{aligned}
$$

This solution is exactly the same as in the "ordinary" non-relativistic EFT (without sea and ghost sectors), with $\alpha, \beta, \gamma$ being the physical $K$-matrix elements, which we are aiming to extract from the lattice data. Note that the physical matrix elements do not depend on the unphysical entry $\delta$.

To summarize, in the infinite volume the solutions of the LS equation of the nonrelativistic EFT with valence, sea and ghost sectors coincide with those in the theory with the valence quarks only. In order to prove this statement, it was crucial to use the symmetry relations between various physical and non-physical $T$-matrix matrix elements, which are given eqs. (3.8), (3.11) and (3.12). This result, of course, was expected from the beginning, since these two theories are equivalent in the infinite volume.

\section{Derivation of the partially twisted Lüscher equation}

Establishing the symmetries of the potential $V$ was the most difficult part of the problem. After this, the derivation of the partially twisted Lüscher equation is straightforward. The prescription, which allows one to get the finite-volume spectrum from the Lüscher equation is to replace the free Green function $G$ by its finite-volume counterpart. Different boundary conditions will lead to the different modifications of $G$. On the contrary, the potential $V$, which encodes the short-range physics, stays unaffected (up to exponentially suppressed contributions).

Let us consider various scenarios and see in detail, how this prescription works. 
Scenario 1. We impose periodic boundary conditions on the $u$-, $d$-quarks and twisted boundary conditions on the $s$-quark:

$$
u(\mathbf{x}+\mathbf{n} L)=u(\mathbf{x}), \quad d(\mathbf{x}+\mathbf{n} L)=d(\mathbf{x}), \quad s(\mathbf{x}+\mathbf{n} L)=e^{i \boldsymbol{\theta} \mathbf{n}} s(\mathbf{x}) .
$$

These boundary conditions translate into the boundary conditions for the meson states: the pions, etas and $\eta^{s}$ fields obey periodic boundary conditions, whereas the boundary conditions for the kaons change:

$K^{ \pm}(\mathbf{x}+\mathbf{n} L)=e^{\mp i \boldsymbol{\theta} \mathbf{n}} K^{ \pm}(\mathbf{x}), K^{0}(\mathbf{x}+\mathbf{n} L)=e^{-i \boldsymbol{\theta} \mathbf{n}} K^{0}(\mathbf{x}), \bar{K}^{0}(\mathbf{x}+\mathbf{n} L)=e^{i \boldsymbol{\theta} \mathbf{n}} \bar{K}^{0}(\mathbf{x})$.

This means that $K$ and $\bar{K}$ mesons containing valence and ghost s-quarks get additional 3 -momenta $\mp \boldsymbol{\theta} / L$. The system stays in the CM frame.

The modified Green function takes the form (cf. with eq. (3.14))

$$
\hat{G}^{L}=\left(\begin{array}{ccccccccc}
B_{L}+\frac{3}{4} Y_{L} & -\frac{1}{4} Y_{L} & -\frac{1}{4} Y_{L} & O_{1} & O_{1} & O_{1} & O_{1} & O_{1} \\
-\frac{1}{4} Y_{L} & -\frac{1}{4} Y_{L} & \frac{1}{4} Y_{L} & O_{1} & O_{1} & O_{1} & O_{1} & O_{1} \\
-\frac{1}{4} Y_{L} & \frac{1}{4} Y_{L} & O_{2} & O_{1} & O_{1} & O_{1} & O_{1} & O_{1} \\
O_{1}^{T} & O_{1}^{T} & O_{1}^{T} & K_{L}^{\theta} & 0 & 0 & 0 & 0 \\
O_{1}^{T} & O_{1}^{T} & O_{1}^{T} & 0 & K_{L} & 0 & 0 & 0 \\
O_{1}^{T} & O_{1}^{T} & O_{1}^{T} & 0 & 0 & -K_{L}^{\theta} & 0 & 0 \\
O_{1}^{T} & O_{1}^{T} & O_{1}^{T} & 0 & 0 & 0 & P_{L} & 0 \\
O_{1}^{T} & O_{1}^{T} & O_{1}^{T} & 0 & 0 & 0 & 0 & -P_{L}
\end{array}\right),
$$

where the substitution rule is (cf. with ref. [9])

$$
\begin{aligned}
K_{L}, E_{L}, P_{L}, S_{L} & =\frac{1}{4 \pi^{3 / 2} P_{0} L} Z_{00}\left(1 ; q^{2}\right), \\
K_{L}^{\theta} & =\frac{1}{4 \pi^{3 / 2} P_{0} L} Z_{00}^{\theta}\left(1 ; q^{2}\right), \quad q=\frac{p L}{2 \pi} .
\end{aligned}
$$

Here, $Z_{00}\left(Z_{00}^{\theta}\right)$ denotes the (twisted) Lüscher zeta-function

$$
\begin{aligned}
& Z_{00}\left(1 ; q^{2}\right)=\frac{1}{\sqrt{4 \pi}} \sum_{\mathbf{n} \in \mathbb{Z}^{3}} \frac{1}{\mathbf{n}^{2}-q^{2}} \\
& Z_{00}^{\theta}\left(1 ; q^{2}\right)=\frac{1}{\sqrt{4 \pi}} \sum_{\mathbf{n} \in \mathbb{Z}^{3}} \frac{1}{(\mathbf{n}+\boldsymbol{\theta} / 2 \pi)^{2}-q^{2}} .
\end{aligned}
$$

In the above equation, an ultraviolet regularization (e.g., the analytic regularization) is implicit. The free Green function in a finite volume, $\hat{G}_{L}$, can be again split in analogy with 
eq. (3.17). The crucial point here is that the symmetry of the $\hat{G}_{1 L}$, which is the finitevolume counterpart of $\hat{G}_{1}$, remains the same. Consequently, the relations in eq. (3.19) still hold in a finite volume. Taking into account this fact, we can rewrite the LS equation (3.21) in a finite volume:

$$
\tau_{L}=\sigma_{L}+\sigma_{L} g_{L} \tau_{L}
$$

where

$$
g_{L}=\operatorname{diag}\left(E_{L}, K_{L}^{\theta}, K_{L},-K_{L}^{\theta}\right)
$$

and $\sigma_{L}$ is obtained from $\sigma$ through the replacement $\delta \rightarrow \delta_{L}$. Other entries in the matrix $\sigma$, which do not contain contributions from the $\pi \pi$ and $\pi \eta^{s}$ loops, stay volume-independent.

The Lüscher equation takes the form

$$
d_{L}=\operatorname{det}\left(1-\sigma_{L} g_{L}\right)=\left(1-\alpha K_{L}-\gamma E_{L}+\left(\alpha \gamma-\beta^{2}\right) K_{L} E_{L}\right)\left(1-\left(\alpha-\delta_{L}\right) K_{L}^{\theta}\right)^{2}=0 .
$$

It is immediately seen that the determinant vanishes for those energies which obey one of the equations

$$
\begin{aligned}
& 0=1-\alpha K_{L}-\gamma E_{L}+\left(\alpha \gamma-\beta^{2}\right) K_{L} E_{L}, \\
& 0=1-\left(\alpha-\delta_{L}\right) K_{L}^{\theta} .
\end{aligned}
$$

The first equation is identical to the Lüscher equation with no twisting. It does not depend on the non-physical entry $\delta_{L}$. The second equation depends on the twisting angle and contains $\delta_{L}$. Since unphysical quantities appear, this equation is not very useful for the analysis of the data.

As seen, the spectrum of the partially twisted equation contains more states than the fully twisted one. Choosing particular source/sink operators, which do not have an overlap with some of the states, one may project out a part of the spectrum. For example, in our case we may consider the quark-antiquark scalar operator $\mathcal{O}_{s}=\bar{u} d$, or the 4-quark operator producing $\pi \eta$ scattering state $\mathcal{O}_{\pi \eta}=\left(\bar{u} \gamma_{5} d\right)\left(\bar{u} \gamma_{5} u+\bar{d} \gamma_{5} d-2 \bar{s} \gamma_{5} s\right) / \sqrt{6}$. It is clear that the spectrum, "seen" by these operators, does not depend on the twisting angle $\boldsymbol{\theta}$. Consequently, these operators do not overlap with the part of the spectrum, described by the second equation in eq. (4.9). At the level of the EFT, this is verified, e.g, from the fact that the $\pi \eta$ scattering amplitude

$$
\left(\tau_{L}\right)_{11}=\frac{\gamma-\left(\alpha \gamma-\beta^{2}\right) K_{L}}{1-\alpha K_{L}-\gamma E_{L}+\left(\alpha \gamma-\beta^{2}\right) K_{L} E_{L}},
$$

has poles, emerging only from the first equation in eq. (4.9) (note that, say, the $K \bar{K}$ amplitude, which is the solution of the same LS equation in a finite volume, contains all poles from eq. (4.9)).

To summarize, it is possible to derive the Lüscher equation with a partially twisted $s$ quark. The spectrum is dependent on the choice of the source/sink operators. Choosing the operators that do not have overlap on the unphysical part of the spectrum, it is seen that the remaining energy levels can be analyzed by using the Lüscher equation with no twisting at all. This is not interesting, because imposing partially twisted boundary condition does not yield new information in this case. 
Scenario 2. Here we consider twisting of the $u$-quark, leaving the $d$ - and $s$-quarks to obey periodic boundary conditions. What changes here is the free Green function in a finite volume.

$$
K, E, P, S \rightarrow K_{L}^{\theta}, E_{L}^{\theta}, P_{L}^{\theta}, S_{L}^{\theta}=\frac{1}{4 \pi^{3 / 2} \sqrt{s} \gamma L} Z_{00}^{\mathrm{d}}\left(1 ;\left(q^{*}\right)^{2}\right)
$$

where $\mathbf{d}=\mathbf{P} L / 2 \pi=\boldsymbol{\theta} / 2 \pi, s=P_{0}^{2}-\mathbf{P}^{2}, \gamma=P_{0} / \sqrt{s}$, and

$$
q^{*}=\frac{p^{*} L}{2 \pi}, \quad p^{*}=\frac{\lambda^{1 / 2}\left(s, m_{1}^{2}, m_{2}^{2}\right)}{2 \sqrt{s}} .
$$

The quantity $Z_{00}^{\mathrm{d}}\left(1 ;\left(q^{*}\right)^{2}\right)$ denotes the Lüscher zeta-function in the moving frame [50], see also refs. [43, 51]:

$$
\begin{aligned}
Z_{00}^{\mathbf{d}}\left(1 ;\left(q^{*}\right)^{2}\right) & =\frac{1}{\sqrt{4 \pi}} \sum_{\mathbf{r} \in P_{d}} \frac{1}{\mathbf{r}^{2}-\left(q^{*}\right)^{2}}, \\
P_{d} & =\left\{\mathbf{r}=\mathbb{R}^{3} \mid r_{\|}=\gamma^{-1}\left(n_{\|}-\mu_{1}|\mathbf{d}|\right), \mathbf{r}_{\perp}=\mathbf{n}_{\perp}, \mathbf{n} \in \mathbb{Z}^{3}\right\},
\end{aligned}
$$

where $\mu_{1}=\left(1-\left(m_{1} 2-m_{2} 2\right) / s\right) / 2$. Here, we would like to mention that, in this scenario, $\pi^{+}$(particle 2 in our nomenclature) is twisted in the propagators $E_{L}^{\theta}$, $S_{L}^{\theta}$, whereas $\eta, \eta^{s}$ (particle 1) are subject to the periodic boundary conditions. This can be easily understood, analyzing the quark diagrams for the different intermediate states given in table 1 . For $K_{L}^{\theta}, P_{L}^{\theta}$, either particle can be twisted since both particles in the intermediate state have the same mass.

The solution of the Lippmann-Schwinger equation in a finite volume takes the following form (cf. with eq. (3.24))

$$
\begin{aligned}
a_{L} & =\frac{\alpha-E_{L}^{\theta}\left(\alpha \gamma-\beta^{2}\right)}{D_{L}^{\theta}}, \quad b_{L}=\frac{\beta}{D_{L}^{\theta}}, \quad c_{L}=\frac{\gamma-K_{L}^{\theta}\left(\alpha \gamma-\beta^{2}\right)}{D_{L}^{\theta}}, \\
D_{L}^{\theta} & =\left(1-K_{L}^{\theta} \alpha\right)\left(1-E_{L}^{\theta} \gamma\right)-K_{L}^{\theta} E_{L}^{\theta} \beta^{2} .
\end{aligned}
$$

It is seen that the spectra in case of the partial and full twisting coincide. Both 4-quark and quark-antiquark operators couple to those eigenstates, whose energies are described by the Lüscher equation in the moving frame

$$
D_{L}^{\theta}=0
$$

Summary. Other scenarios are possible. For example, one may consider twisting $u$ - and $d$-quarks with the same angle, in order to bring two particles again in the CM frame. We do not consider more scenarios in detail, since the pattern is already clear from the above examples.

One observes that there exists the rule of thumb for the scenarios considered above. Namely, if in a given scenario the twisted valence quarks may annihilate (as in the scenario 1 ), then the corresponding partial twisting will effectively yield no twisting. On the other hand, if the twisted valence quarks "go through" all diagrams without annihilating (as in 
the scenario 2), then the partially twisted Lüscher equation is equivalent to the fully twisted one up to exponentially suppressed terms. The first case is indeed easy to understand without doing any calculations: for studying the spectrum, one could use, for example, the quark-antiquark source and sink operators $\bar{u} d, \bar{d} u$, which do not change at all, when the valence $s$-quarks are twisted. The result in the second case looks also plausible, when one considers quark diagrams, corresponding to the two-particle scattering processes. However, due to technical complications, arising mainly from the neutral meson mixing, certain effort is needed to elevate a plausible statement to a proof.

\section{Meson mixing in the neutral sector}

In the preceding sections we have derived symmetry relations for the elements of the scattering $T$-matrix, assuming the exact $\mathrm{SU}(3)$-symmetric quark content of the states corresponding to the $\eta, \eta^{\prime}$ mesons: $\eta=\eta^{8} \sim(u \bar{u}+d \bar{d}-2 s \bar{s}) / \sqrt{6}$ and $\eta^{\prime}=\eta^{0} \sim(u \bar{u}+d \bar{d}+s \bar{s}) / \sqrt{3}$ in the valence, sea and ghost quark sectors (note that not all of these states are independent due to the condition $\operatorname{str} \Phi=0$ ). This assumption holds only, if $m_{s}=\hat{m}$. At the level of the EFT, described by the Lagrangian in eq. (2.6), the above relations hold at tree level and are broken by $O\left(p^{4}\right)$ corrections. Do our results, which rely heavily on the symmetry relations, survive, if the mixing is taken into account to all orders?

The answer to this question is positive. The physical justification of this fact is very transparent: in the derivation of the symmetry relations itself that involved the comparison of the quark diagrams (see section 3), we have never required $m_{s}=\hat{m}$. Rather, it was assumed that the masses of the valence, sea and ghost quarks for each flavor coincide (this requirement is fulfilled in our case). So, one expects that the results are not affected by the breaking of the flavor $\mathrm{SU}(3)$.

To elevate this argument to a formal level, let us consider the two-point function of two quark bilinears in the EFT

$$
D_{i j}\left(p^{2}\right)=i \int d x e^{i p x}\left\langle 0\left|T \chi_{i}(x) \chi_{j}(0)\right| 0\right\rangle, \quad i, j=1, \cdots, 6,
$$

where $\chi_{i}=\bar{\psi} \Gamma_{i} \psi$ and the matrices $\Gamma_{i}$ carry all information about the spin-flavor content of the mesons. For our goals, it suffices to consider $\eta, \eta^{\prime}$ mesons only (the pions and kaons do not mix). The fermions $\psi, \bar{\psi}$ belong to either valence, sea or ghost sectors.

The pole structure of $D_{i j}\left(p^{2}\right)$ is given by

$D_{i j}\left(p^{2}\right)=\sum_{\alpha=1}^{6} \Lambda_{i \alpha} D_{\alpha}\left(p^{2}\right) \Lambda_{\alpha j}^{T}+D_{i j}\left(p^{2}\right)^{\text {non }- \text { pole }}, \quad D_{\alpha}\left(p^{2}\right)=\frac{c_{\alpha}}{M_{\alpha}^{2}-p^{2}}, \quad c_{\alpha}= \pm 1,0$

where, at tree level, the elements of the matrix, up to a common normalization, $\Lambda_{i \alpha}$, can be read off from eq. (2.11). These matrix elements get modified at higher orders in ChPT, if the flavor $\mathrm{SU}(3)$ is broken through $\hat{m} \neq m_{s}$. The masses $M_{\alpha}^{2}=M_{\pi}^{2}, M_{\eta}^{2}, M_{s}^{2}$ are all equal in the $\mathrm{SU}(3)$ symmetry limit. At $O\left(p^{2}\right)$, their values can be read off from eq. (2.12). 
The matrix $\Lambda$, which relates the meson fields in the $\mathrm{SU}(3)$ and physical bases, can be written in the following form:

$$
\Lambda_{i \alpha}=\sum_{m=1}^{6} \tilde{\Lambda}_{i m} \stackrel{0}{\Lambda}_{m \alpha}
$$

where $\stackrel{0}{\Lambda}_{m \alpha}$ denotes the matrix at $O\left(p^{2}\right)$ (so far, we have worked with this matrix), and $\tilde{\Lambda}_{i m}$ collects all higher-order corrections.

Let us now consider the 4-point function of the quark bilinears, corresponding to the $\pi^{+} \eta\left(\eta^{\prime}\right) \rightarrow \pi^{+} \eta\left(\eta^{\prime}\right)$ scattering, see the table 1 ,

$$
\begin{aligned}
& (2 \pi)^{4} \delta^{4}\left(p_{1}+p_{2}-q_{1}-q_{2}\right) \Gamma_{i j}\left(p_{1}, p_{2} ; q_{1}, q_{2}\right) \\
= & \int d x_{1} d x_{2} d y_{1} d y_{2} e^{i p_{1} x_{1}+i p_{2} x_{2}-i q_{1} y_{1}-i q_{2} y_{2}}\left\langle 0\left|T \chi_{i}\left(x_{1}\right) \chi_{\pi^{+}}\left(x_{2}\right) \chi_{j}\left(y_{1}\right) \chi_{\pi^{-}}\left(y_{2}\right)\right| 0\right\rangle
\end{aligned}
$$

The connected piece of the 4-point function can be written as

$$
\begin{aligned}
& \Gamma_{i j}\left(p_{1}, p_{2} ; q_{1}, q_{2}\right)^{\mathrm{conn}} \\
= & \sum_{k l} D_{i k}\left(p_{1}^{2}\right) D_{\pi^{+}}\left(p_{2}^{2}\right) T_{k l}\left(p_{1}, p_{2} ; q_{1}, q_{2}\right) D_{\pi^{+}}\left(q_{2}^{2}\right) D_{l j}\left(q_{1}^{2}\right) \\
= & \sum_{k l} \sum_{\alpha \beta} \Lambda_{i \alpha} D_{\alpha}\left(p_{1}^{2}\right) \Lambda_{\alpha k}^{T} D_{\pi^{+}}\left(p_{2}^{2}\right) T_{k l}\left(p_{1}, p_{2} ; q_{1}, q_{2}\right) D_{\pi^{+}}\left(q_{2}^{2}\right) \Lambda_{l \beta} D_{\beta}\left(q_{1}^{2}\right) \Lambda_{\beta j}^{T}+\cdots
\end{aligned}
$$

From the above expression it is clear that the scattering amplitude in the "physical" basis (i.e., the basis which diagonalizes the matrix of the two-point functions), on the mass shell is given by

$$
\begin{aligned}
& T_{\alpha \beta}^{\text {on-shell }}(s, t)=\lim _{p_{1}^{2} \rightarrow M_{\alpha}^{2}, q_{1}^{2} \rightarrow M_{\beta}^{2}, p_{2}^{2}, q_{2}^{2} \rightarrow M_{\pi}^{2}} T_{\alpha \beta}\left(p_{1}, p_{2} ; q_{1}, q_{2}\right) \\
&=p_{1}^{2} \rightarrow M_{\alpha}^{2}, q_{1}^{2} \rightarrow M_{\beta}^{2}, p_{2}^{2}, q_{2}^{2} \rightarrow M_{\pi}^{2} \\
& \Lambda_{\alpha k}^{T} T_{k l}\left(p_{1}, p_{2} ; q_{1}, q_{2}\right) \Lambda_{l \beta},
\end{aligned}
$$

where $s, t$ are the usual Mandelstam variables.

Now, let us consider the situation that the 4-point function of the quark-antiquark bilinears obeys some symmetry relations (an analogy of the relations considered in the section 3 )

$$
\sum_{i j} d_{j i} \Gamma_{i j}\left(p_{1}, p_{2} ; q_{1}, q_{2}\right)=0
$$

where $d_{i j}$ are some numerical coefficients related to the structure of the symmetry group (but not to the dynamics). Note that these are relations that hold for off-shell momenta $p_{1}, p_{2}, q_{1}, q_{2}$.

One has to further distinguish between the case of the exact $\mathrm{SU}(3)$ flavor symmetry and broken $\mathrm{SU}(3)$ flavor symmetry. 
Exact SU(3) symmetry. In this case $\Lambda={ }_{\Lambda}^{0}$ exactly, to all orders in ChPT. Further, substituting eq. (5.5) into eq. (5.7) and performing the mass-shell limit, we get

$$
\sum_{\alpha \beta} k_{\beta \alpha} T_{\alpha \beta}^{\text {on-shell }}(s, t)=0, \quad k_{\beta \alpha}=\sum_{i j} \Lambda_{\beta j}^{T} d_{j i} \Lambda_{i \alpha}
$$

We remind the reader that the disconnected piece does not have four poles in the external momenta squared.

Next we define the on-shell amplitudes in the SU(3) basis

$$
T_{i j}^{\text {on-shell }}(s, t)=\sum_{\alpha \beta} \Lambda_{i \alpha} T_{\alpha \beta}^{\text {on-shell }}(s, t) \Lambda_{\beta j}^{T}, \quad \sum_{i j} d_{j i} T_{i j}^{\text {on-shell }}(s, t)=0 .
$$

In other words, in case of exact SU(3) symmetry, the symmetry relations on the 4-point functions directly translate in the relations for the on-shell amplitudes.

The LS equation in the non-relativistic EFT is derived in the basis where the two-point function is diagonal (see section 2). This (matrix) equation can be written in the form

$$
T_{\alpha \beta}^{\mathrm{on}-\text { shell }}=V_{\alpha \beta}+\sum_{\gamma} V_{\alpha \gamma} G_{\gamma} T_{\gamma \beta}^{\mathrm{on}-\text { shell }}
$$

where the $G_{\gamma}$ are loops ${ }^{7}$ with the $\pi^{+}$and the "particle" $\gamma$. Changing now to the SU(3) basis, we arrive at the equation

$$
T_{i j}^{\mathrm{on}-\text { shell }}=V_{i j}+\sum_{n m} V_{i n} G_{n m} T_{m j}^{\mathrm{on}-\text { shell }}
$$

where the relation between $V_{i j}$ and $V_{\alpha \beta}$ is the same as between $T_{i j}^{\text {on-shell }}$ and $T_{\alpha \beta}^{\text {on-shell }}$, and the free Green function in the new basis is given by

$$
G_{i j}=\sum_{\gamma}\left(\Lambda^{T}\right)_{i \gamma}^{-1} G_{\gamma} \Lambda_{\gamma j}^{-1}
$$

Our equations given in section 3 are exactly reproduced. The derivation of the Lüscher equation is straightforward. All results remain valid.

Broken SU(3) symmetry. In Nature, $\hat{m} \neq m_{s}$. One may still have some exact relations of the type given in eq. (5.7) - those, which do not require $\hat{m}=m_{s}$. Examples of such relations are given in section 3 .

There are five neutral one-particle states with isospin $I=0$ in the physical basis (cf. with eq. (2.14)). These states belong to the three different classes. Namely, there is one state with $M_{\alpha}^{2}=M_{\eta}^{2}$, two states (one with the wrong sign in the kinetic term) with $M_{\alpha}^{2}=M_{s}^{2}$ and two states (one with the wrong sign in the kinetic term) with $M_{\alpha}^{2}=M_{\pi}^{2}$. In eq. (2.14), these states are described by the fields $\omega_{2}$ and $\omega_{3}, \omega_{6}$ and $\omega_{5}, \omega_{8}$, respectively (of course, the numerical values of the coefficients in this equation are different from the

\footnotetext{
${ }^{7}$ For simplicity, we neglect here the part of the free Green function, which is already diagonal, e.g., the $K \bar{K}$ loops. Taking them into account does not change anything in our argumentation.
} 
$O\left(p^{2}\right)$ values given in eq. (2.14)). We introduce a special notation for the above classes $M=\eta, \eta^{s}, \pi$.

Let us now consider eq. (5.7) in the vicinity of the poles in the momenta of the external particles. Since the masses of the particles, belonging to the different classes, differ, if $\hat{m} \neq m_{s}$, the residues should vanish independently for each class. Consequently,

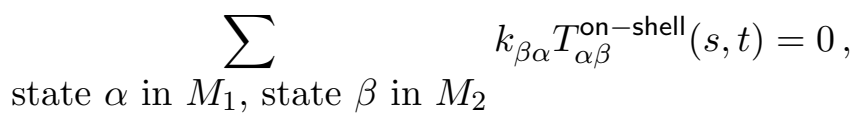

where the sum runs only over those states which belong to the classes $M_{1}$ and $M_{2}$, respectively. For example, if $M_{1}=M_{2}=\eta$, from the above equation we get: $k_{22} T_{22}^{\text {on-shell }}=0$. If $M_{1}=\eta$ and $M_{2}=\eta^{s}$, we get $k_{32}\left(T_{23}^{\text {on-shell }}+T_{32}^{\text {on-shell }}\right)+k_{62}\left(T_{26}^{\text {on-shell }}+T_{62}^{\text {on-shell }}\right)=0$, and so on (here, we have used the fact that the matrix $T_{\alpha \beta}^{\text {on-shell }}$ is symmetric, as well as the $\left.\operatorname{matrix} k_{\alpha \beta}\right)$.

Now, let us define

$$
\stackrel{0}{k}_{\beta \alpha}=\sum_{i j} \stackrel{0}{\Lambda}_{\beta j}^{T} d_{j i} \stackrel{0}{\Lambda}_{i \alpha} .
$$

The following crucial statement is proven in the appendix B:

There is certain freedom in choosing the quantities $d_{i j}$. For example, if we have two independent linear relations between $\Gamma_{i j}$, adding these relations with arbitrary coefficients will yield a relation as well. Using this freedom, one may choose the quantities $d_{i j}$ so that the following relation holds separately for each $M_{1}, M_{2}$

$$
k_{\beta \alpha}=h\left(M_{1}, M_{2}\right) \stackrel{0}{k_{\beta \alpha}} .
$$

Here, $\alpha, \beta$ label the states in the classes $M_{1}, M_{2}$, respectively, and the number $h\left(M_{1}, M_{2}\right)$ does not depend on $\alpha$ and $\beta$.

The rest of the proof is straightforward. We define the $T$-matrix in the $\mathrm{SU}(3)$ basis through

$$
T_{i j}^{\text {on-shell }}(s, t)=\sum_{\alpha \beta} \stackrel{0}{\Lambda}_{i \alpha} T_{\alpha \beta}^{\text {on-shell }}(s, t) \stackrel{0}{\Lambda}_{\beta j}^{T} .
$$

We would like to stress that this is merely a definition, which is made for mathematical convenience only. Physically, it does not make sense to consider a superposition of the states with different masses in case of broken SU(3) symmetry.

Using eqs. (5.13) and (5.15), we easily derive a counterpart of eq. (5.8)

$$
\sum_{\alpha \beta} \stackrel{0}{k}_{\beta \alpha} T_{\alpha \beta}^{\text {on-shell }}(s, t)=0,
$$

where the sum now runs over all $\alpha, \beta$ from different classes $M_{1}, M_{2}$. 
Finally, for the above definition of the $T$-matrix, one gets

$$
\sum_{i j} d_{j i} T_{i j}^{\text {on-shell }}(s, t)=0
$$

The free Green function in the LS equation is given by

$$
G_{i j}=\sum_{\gamma}\left(0^{T}\right)_{i \gamma}^{-1} G_{\gamma}(\stackrel{0}{\Lambda})_{\gamma j}^{-1},
$$

and we arrive exactly at the same expressions as before. The derivation of the Lüscher equation is again straightforward, since only neutral mesons with the isospin $I=0$ are affected by the mixing. The crucial point is that there is no effect of twisting for these mesons because they are neutral. Consequently, no ambiguity arises in the construction of the free Green function in the partially twisted case.

To summarize, the SU(3) breaking affects both the free Green function and the scattering amplitude. The matrix $\Lambda_{i \alpha}$ differs from its $O\left(p^{2}\right)$ value $\stackrel{0}{\Lambda}_{i \alpha}$. It is, however, possible to define the free Green function and the $T$-matrix in the $\mathrm{SU}(3)$ basis, still using the matrix $\stackrel{0}{\Lambda}_{i \alpha}$, even if $\hat{m} \neq m_{s}$. It can be now checked explicitly that the symmetry relations from section 3 hold for the elements of the T-matrix in the SU(3) basis. Consequently, our final results are unaffected by $\mathrm{SU}(3)$ breaking. This was, of course, expected from the beginning, since the LS equation - with the use of the above-mentioned symmetry relations should reduce to the one in the valence sector only in the infinite volume even in case of $\hat{m} \neq m_{s}$.

\section{Conclusions and outlook}

i) Using the non-relativistic EFT technique in a finite volume, we have derived the Lüscher equation for the partially twisted boundary conditions for coupled-channel $\pi \eta-K \bar{K}$ scattering. At an intermediate step, the matching of the non-relativistic Lagrangian to partially quenched ChPT has been considered.

ii) Our final result is remarkably simple. If in the channel with $I=I_{3}=1$ the light quarks are subject to twisting, the partially twisted Lüscher equation is equivalent to the fully twisted one, despite the presence of annihilation diagrams. If, on the contrary, partial twisting of the strange quark is performed, the physically interesting part of the spectrum is not affected. Other scenarios are also possible and can be investigated by using the same methods. We think that this result is interesting for the lattice practitioners studying the properties of scalar mesons. We have shown that, instead of carrying out simulations at different volumes, as required in the Lüscher approach, one may perform relatively cheaper partially twisted simulations.

iii) In order to demonstrate the above result, one relies heavily on the relations that emerge between the various $T$-matrix elements from the valence, sea and ghost sectors of the theory, and stem from the fact that the masses of the valence, sea and 
ghost quarks are taken equal. These relations lead to numerous cancellations in the LS equation, so that in the final equation only the physical amplitudes, i.e., the amplitudes from the valence quark sector, are present. There are strong intuitive arguments, which support the above statement. However, due to the techical complications, owing mainly to the neutral meson mixing, a certain effort was still needed to transform these arguments into a valid proof.

iv) We have carried out the derivation within certain approximations. For example, we consider only the channel with total isospin $I=1$. Moreover, all partial waves except $l=0$ are neglected from the beginning. The partial-wave mixing can be included later by using standard techniques (see, e.g., refs. [51-53]). Here, our aim was to describe the method in the most transparent manner for one particular example, without overloading the arguments with inessential details. Further, the method described above can be used in other systems as well, for example, in the study of the $D K$ molecules in lattice QCD (the work on this problem is in progress, and the results will be reported elsewhere).

\section{Acknowledgments}

The authors thank S. Beane, J. Bijnens, J. Gasser, T. Lähde, Ch. Liu, M. Savage, S. Sharpe and C. Urbach for interesting discussions. One of us (AR) thanks the Institute for Nuclear Theory at the University of Washington for its hospitality and the Department of Energy for partial support during the completion of this work. This work is partly supported by the EU Integrated Infrastructure Initiative HadronPhysics3 Project under Grant Agreement no. 283286. We also acknowledge the support by the DFG (CRC 16, "Subnuclear Structure of Matter"), by the DFG and NSFC (CRC 110, "Symmetries and the Emergence of Structure in QCD"), by the Shota Rustaveli National Science Foundation (Project DI/13/02) and by the Bonn-Cologne Graduate School of Physics and Astronomy. This research is supported in part by Volkswagenstiftung under contract no. 86260.

\section{A Explicit form of the matrices $T_{j}$ in eq. (2.13)}

In this appendix we give an explicit form of the matrices $T_{j}$ which appear in eq. (2.13)

$$
\begin{aligned}
T_{1} & =\frac{1}{\sqrt{2}} \operatorname{diag}(1,-1,0,0,0,0,0,0,0) \\
T_{2} & =\frac{1}{\sqrt{6}} \operatorname{diag}(-1,-1,2,-1,-1,2,-1,-1,2), \\
T_{3} & =\frac{1}{\sqrt{2}} \operatorname{diag}(0,0,1,0,0,1,0,0,2) \\
T_{4} & =\frac{1}{\sqrt{2}} \operatorname{diag}(0,0,0,1,-1,0,0,0,0) \\
T_{5} & =\frac{1}{2} \operatorname{diag}(-1,-1,0,1,1,0,0,0,0)
\end{aligned}
$$




$$
\begin{aligned}
T_{6} & =\frac{1}{\sqrt{2}} \operatorname{diag}(0,0,-1,0,0,1,0,0,0), \\
T_{7} & =\frac{1}{\sqrt{2}} \operatorname{diag}(0,0,0,0,0,0,1,-1,0), \\
T_{8} & =\frac{1}{2} \operatorname{diag}(1,1,0,1,1,0,2,2,0) .
\end{aligned}
$$

\section{B Proof of eq. (5.15)}

\section{B.1 The structure of the matrix $\Lambda_{i \alpha}$}

The quantity $D_{i j}$ in eq. (5.1) is a $6 \times 6$ matrix. containing correlators of the quark bilinears $\eta_{\mathrm{vv}}^{8}, \eta_{\mathrm{vv}}^{0}, \eta_{\mathrm{ss}}^{8}, \eta_{\mathrm{ss}}^{0}, \eta_{\mathrm{gg}}^{8}, \eta_{\mathrm{gg}}^{0}$ where, for example, $\eta_{\mathrm{vv}}^{8}=\left(\bar{u}_{\mathrm{v}} u_{\mathrm{v}}+\bar{d}_{\mathrm{v}} d_{\mathrm{v}}-2 \bar{s}_{\mathrm{v}} s_{\mathrm{v}}\right) / \sqrt{6}$ and so on. Consider now the quark diagrams describing the two-point function of the quark bilinears, see figure B.4. The diagonal matrix elements contain both connected and disconnected pieces. Keeping track of the signs emerging in the result of (anti)commuting the fields, we get

$$
D_{\mathrm{vv}}=D_{\mathrm{ss}}=-z_{c}+z_{d}, \quad D_{\mathrm{gg}}=z_{c}+z_{d}
$$

Here, all quantities are $2 \times 2$ matrices.

The non-diagonal matrix elements contain only the disconnected piece:

$$
D_{\mathrm{vs}}=D_{\mathrm{sg}}=D_{\mathrm{gv}}=z_{d} .
$$

Taking into account the above formulae, one may conclude that the matrix $D_{i j}$ has, in general, the following structure (cf. with eq. (2.19) $)^{8}$

$$
D_{i j}=\left(\begin{array}{ccc}
\hat{A} & \hat{A}+\hat{X} & \hat{A}+\hat{X} \\
\hat{A}+\hat{X} & \hat{A} & \hat{A}+\hat{X} \\
\hat{A}+\hat{X} & \hat{A}+\hat{X} & \hat{A}+2 \hat{X}
\end{array}\right), \quad \hat{A}=-z_{c}+z_{d}, \quad \hat{X}=z_{c} .
$$

Here, $\hat{A}, \hat{X}$ are $2 \times 2$ matrices.

The quantity $\hat{A}$ in the upper left corner of the matrix $D$ is the physical propagator (it contains only valence quarks). Consequently, it has only a pole at $p^{2} \rightarrow M_{\eta}^{2}$. In the vicinity of the pole,

$$
\hat{A}_{i j}\left(p^{2}\right) \rightarrow \frac{\Lambda_{i \alpha} \Lambda_{\alpha j}^{T}}{M_{\eta}^{2}-p^{2}}+\text { regular terms, } \quad i, j=1,2, \quad \text { state } \alpha \text { in } \eta
$$

\footnotetext{
${ }^{8}$ The general structure of the two-point function in the partially quenched ChPT has been discussed, e.g., in ref. [54]
} 

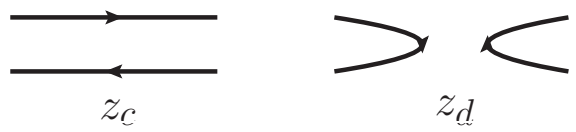

Figure B.4. Quark diagrams for the two-point function of two quark bilinears. There are connected, $z_{c}$ and disconnected, $z_{d}$ contributions in the diagonal matrix elements. Non-diagonal matrix elements contain only disconnected contribution.

Following the nomenclature of eq. (2.14), the state in the class $\eta$ corresponds to $\alpha=2$. Further, choosing the proper normalization, we may write

$$
\Lambda_{1 \alpha}=\cos \tilde{\theta} \doteq c, \quad \Lambda_{2 \alpha}=\sin \tilde{\theta} \doteq s, \quad \text { state } \alpha \text { in } \eta .
$$

We shall call $\tilde{\theta}$ the mixing angle. The equation $(2.20)$ corresponds to $\tilde{\theta}=0$.

Now, let us prove that the quantity $\hat{X}$ does not have a pole at $p^{2} \rightarrow M_{\eta}^{2}$. To this end, note that the residue at the pole should be separable. Consequently, the $2 \times 2$ matrices $\hat{A}, \hat{A}+\hat{X}, \hat{A}+2 \hat{X}$ are all separable in the vicinity of $p^{2}=M_{\eta}^{2}$. Since a separable matrix has one vanishing eigenvalue, with a orthogonal transformation $O$ the matrix $\hat{A}$ can be brought to the diagonal form $O \hat{A} O^{T}=\operatorname{diag}(\lambda, 0)$. Further, since the determinant of a separable matrix vanishes, we have: $\operatorname{det}(\hat{A}+\hat{X})=\operatorname{det}(\hat{A}+2 \hat{X})=0$ in the vicinity of the pole. Recalling that the matrix $\hat{X}$ is symmetric, it can be explicitly checked that this condition can be fulfilled, if and only if $O \hat{X} O^{T}=\operatorname{diag}\left(\lambda^{\prime}, 0\right)$, i.e., $\hat{X}=\mathcal{N} \hat{A}$ in the vicinity of the pole.

The value of the constant $\mathcal{N}$ can be fixed through the following argument. One may change the basis $\eta^{8}, \eta^{0}$ to $\eta^{l}=(\bar{u} u+\bar{d} d) / \sqrt{2}$ and $\eta^{s}=\bar{s} s$, in valence, sea and ghost sectors. The matrix $D_{i j}$ in the new basis has the same general structure as before. Further, it is immediately seen that certain diagonal and non-diagonal matrix elements are equal (both contain only disconnected contributions). For example,

$$
\left\langle 0\left|T \eta_{\mathrm{vv}}^{l}(x) \eta_{\mathrm{vv}}^{s}(y)\right| 0\right\rangle=\left\langle 0\left|T \eta_{\mathrm{vv}}^{l}(x) \eta_{\mathrm{ss}}^{s}(y)\right| 0\right\rangle
$$

Considering the limit $p^{2} \rightarrow M_{\eta}^{2}$, one may check that the above condition is fulfilled, if and only if $\mathcal{N}=0$. In other words, the matrix $\hat{X}$ does not have a pole at $p^{2} \rightarrow M_{\eta}^{2}$.

Next, we wish to demonstrate that there is no mixing, when $p^{2} \rightarrow M_{s}^{2}$ or $p^{2} \rightarrow M_{\pi}^{2}$. To this end, it is again convenient to use the basis $\eta^{l}, \eta^{s}$ instead of $\eta^{8}, \eta^{0}$. Consider, for example, the case $p^{2} \rightarrow M_{s}^{2}$. Near the pole,

$$
D_{i j}^{\prime}\left(p^{2}\right) \rightarrow \sum_{\text {state } \alpha \text { in } \eta^{s}} \frac{c_{\alpha} \Lambda_{i \alpha}^{\prime} \Lambda_{\alpha j}^{\prime T}}{M_{s}^{2}-p^{2}}+\text { regular terms }, \quad i, j=1,6, \quad c_{\alpha}= \pm 1 .
$$

Here, $D_{i j}^{\prime}$ is obtained from $D_{i j}$ via the orthogonal transformation that corresponds to the change of the basis from $\eta^{8}, \eta^{0}$ to $\eta^{l}, \eta^{s}$. Again following the nomenclature of eq. (2.14), two states in the class $\eta^{s}$ are $\alpha=3$ with $c_{\alpha}=-1$ and $\alpha=6$ with $c_{\alpha}=1$.

It is immediately seen that $\hat{X}_{12}^{\prime}=\hat{X}_{21}^{\prime}=0$ (there are no connected diagrams for the $\eta^{l}-\eta^{s}$ transition). Also, as we shall see below, the pole can be contained either in $\hat{X}_{11}^{\prime}$ 
or in $\hat{X}_{22}^{\prime}$, but not in both. In accordance with eq. (2.20), we assume that the pole is contained in $\hat{X}_{22}^{\prime}$. Taking into account eq. (B.3) and the fact that $\hat{A}^{\prime}$ does not have a pole when $p^{2} \rightarrow M_{s}^{2}$, the following relations hold (up to an overall normalization):

$$
\begin{aligned}
\Lambda_{23}^{\prime 2}-\Lambda_{26}^{\prime 2} & =\Lambda_{43}^{\prime 2}-\Lambda_{46}^{\prime 2}=0, \quad \Lambda_{63}^{\prime 2}-\Lambda_{66}^{\prime 2}=2, \\
\Lambda_{23}^{\prime} \Lambda_{43}^{\prime}-\Lambda_{26}^{\prime} \Lambda_{46}^{\prime} & =\Lambda_{23}^{\prime} \Lambda_{63}^{\prime}-\Lambda_{26}^{\prime} \Lambda_{66}^{\prime}=\Lambda_{43}^{\prime} \Lambda_{63}^{\prime}-\Lambda_{46}^{\prime} \Lambda_{66}^{\prime}=1 .
\end{aligned}
$$

These equations still do not suffice to determine all quantities unambiguously. To proceed further, note that $\Lambda_{2 \alpha}^{\prime}$ and $\Lambda_{4 \alpha}^{\prime}$ describe the coupling of the $\eta_{\mathrm{vv}}^{s}$ and $\eta_{\mathrm{ss}}^{s}$ fields to the state $|\alpha\rangle$ :

$$
\left\langle 0\left|\bar{s}_{\mathrm{v}} s_{\mathrm{v}}\right| \alpha\right\rangle=\Lambda_{2 \alpha}^{\prime}, \quad\left\langle 0\left|\bar{s}_{\mathrm{s}} s_{\mathbf{s}}\right| \alpha\right\rangle=\Lambda_{4 \alpha}^{\prime}
$$

Consequently,

$$
\frac{1}{\sqrt{2}}\left\langle 0\left|\left(\bar{s}_{\mathrm{v}} s_{\mathrm{v}} \pm \bar{s}_{\mathrm{s}} s_{\mathrm{s}}\right)\right| \alpha\right\rangle=\frac{1}{\sqrt{2}}\left(\Lambda_{2 \alpha}^{\prime} \pm \Lambda_{4 \alpha}^{\prime}\right)
$$

Recall now that the operators $\left(\bar{s}_{\mathrm{v}} s_{\mathrm{v}} \pm \bar{s}_{\mathrm{s}} s_{\mathrm{s}}\right) / \sqrt{2}$ transform differently with respect to the horizontal isospin, corresponding to the $\mathrm{SU}(2)$ rotation of the valence quarks into the sea quarks of the same flavor and vice versa. Horizontal isospin is a good quantum number, since the masses of the quarks of different species coincide. The physical states $|\alpha\rangle$ should be characterized by a definite horizontal isospin. This means that both $\left(\bar{s}_{\mathrm{v}} s_{\mathrm{v}}+\bar{s}_{\mathrm{s}} s_{\mathrm{s}}\right) / \sqrt{2}$ and $\left(\bar{s}_{\mathrm{v}} s_{\mathrm{v}}-\bar{s}_{\mathrm{s}} s_{\mathrm{s}}\right) / \sqrt{2}$ can not couple to the same state $|\alpha\rangle$ and, consequently,

$$
\left|\Lambda_{2 \alpha}^{\prime}\right|=\left|\Lambda_{4 \alpha}^{\prime}\right|, \quad \alpha=3,6
$$

With this additional constraint, the above equations have the following solution:

$$
-\Lambda_{23}^{\prime}=\Lambda_{26}^{\prime}=-\Lambda_{43}^{\prime}=-\Lambda_{46}^{\prime}=\frac{1}{\sqrt{2}}, \quad \Lambda_{63}^{\prime}=-\sqrt{2}, \quad \Lambda_{66}^{\prime}=0
$$

Further, since in this basis the non-diagonal matrix elements, corresponding to the $\eta^{l}-\eta^{s}$ transition, do not have a pole, we get

$$
\Lambda_{13}^{\prime}=\Lambda_{16}^{\prime}=\Lambda_{33}^{\prime}=\Lambda_{36}^{\prime}=\Lambda_{53}^{\prime}=\Lambda_{56}^{\prime}=0 \text {. }
$$

We see that the pole can not be contained both in $\hat{X}_{11}^{\prime}$ and $\hat{X}_{22}^{\prime}$. The case of $p^{2} \rightarrow M_{\pi}^{2}$ is treated analogously, only the pole appears now in $\hat{X}_{11}^{\prime}$. Transforming the propagator back to the basis $\eta^{8}, \eta^{0}$, we finally conclude that, up to a normalization of the quantities $D_{s}$ and $D_{\pi}$, the structure of the matrix $\hat{X}$ in the vicinity of the pole is the same as of the matrix $X$ given by eq. (2.20). Consequently, no mixing occurs in the matrix $\hat{X}$.

To summarize, the matrix $\Lambda_{i \alpha}$ has the following structure (the index $i$ runs from 1 to 6$)$ :

- The class $M=\eta$, one state 


$$
\Lambda_{i, \alpha=2} \doteq n_{i}=\left(\begin{array}{c}
c \\
s \\
c \\
s \\
c \\
s
\end{array}\right) .
$$

- The class $M=\eta^{s}$, two states

$$
\Lambda_{i, \alpha=3} \doteq w_{i}^{(1)}=\left(\begin{array}{c}
-\frac{1}{\sqrt{3}} \\
\frac{1}{\sqrt{6}} \\
-\frac{1}{\sqrt{3}} \\
\frac{1}{\sqrt{6}} \\
-\frac{2}{\sqrt{3}} \\
\frac{2}{\sqrt{6}}
\end{array}\right), \quad \Lambda_{i, \alpha=6} \doteq w_{i}^{(2)}=\left(\begin{array}{c}
\frac{1}{\sqrt{3}} \\
-\frac{1}{\sqrt{6}} \\
-\frac{1}{\sqrt{3}} \\
\frac{1}{\sqrt{6}} \\
0 \\
0
\end{array}\right) \text {. }
$$

- The class $M=\pi$, two states

$$
\Lambda_{i, \alpha=5} \doteq \nu_{i}^{(1)}=\left(\begin{array}{c}
-\frac{1}{\sqrt{6}} \\
-\frac{1}{\sqrt{3}} \\
\frac{1}{\sqrt{6}} \\
\frac{1}{\sqrt{3}} \\
0 \\
0
\end{array}\right), \quad \Lambda_{i, \alpha=8} \doteq \nu_{i}^{(2)}=\left(\begin{array}{c}
\frac{1}{\sqrt{6}} \\
\frac{1}{\sqrt{3}} \\
\frac{1}{\sqrt{6}} \\
\frac{1}{\sqrt{3}} \\
\frac{2}{\sqrt{6}} \\
\frac{2}{\sqrt{3}}
\end{array}\right) .
$$

The formulae for $M=\eta^{s}, \pi$ were read off eq. (2.14). The common normalization in each class is unimportant and is omitted. The quantity $\stackrel{0}{\Lambda}_{i \alpha}$ is obtained from $\Lambda_{i \alpha}$ by putting the mixing angle $\tilde{\theta}=0$, i.e., $c=1, s=0$. 


\section{B.2 The linear relations between the four-point functions}

The relation given in eq. (5.15) holds trivially, if $M_{1}=M_{2}=\eta$, since in this case, there is only one state. Moreover, since, as we have found, the structure of $\Lambda_{i \alpha}$ is the same as of $\stackrel{0}{\Lambda}_{i \alpha}$, when $M=\eta^{s}$ or $\pi$, eq. (5.15) also holds, if both $M_{1}$ and $M_{2}$ are either $\eta^{s}$ or $\pi$. What remains to be checked is the case when $M_{1}=\eta$ and $M_{2}=\eta^{s}$ or $\pi$.

Our strategy will be explained in few examples below. Let us start from the identity $f+\tilde{f}=-2 f^{\prime \prime}$, see eq. (3.11). The corresponding (symmetrized) relation for the four-point functions is:

$$
\Gamma_{33}+\Gamma_{55}+\Gamma_{53}+\Gamma_{35}=0
$$

From this, one may read off the coefficients $d_{i j}$

$$
d_{33}=d_{55}=d_{35}=d_{53}=1, \quad d_{i j}=0 \text { otherwise. }
$$

Now, define,

$$
k_{\alpha}^{(s)}=\sum_{i, j=1}^{6} n_{i} w_{j}^{(\alpha)} d_{j i}, \quad k_{\alpha}^{(\pi)}=\sum_{i, j=1}^{6} n_{i} \nu_{j}^{(\alpha)} d_{j i} .
$$

Using the explicit expressions, given in eqs. (B.14), (B.15) and (B.16), we get

$$
k_{1}^{(s)}=-2 c \sqrt{3}, \quad k_{2}^{(s)}=-\frac{2 c}{\sqrt{3}}, \quad k_{1}^{(\pi)}=\frac{2 c}{\sqrt{6}}, \quad k_{2}^{(\pi)}=c \sqrt{6} .
$$

It is clear that eq. (5.15) is fulfilled. The factor $h\left(M_{1}, M_{2}\right)=c$, if $M_{1}=\eta$ and $M_{2}=\eta^{s}$ or $\pi$.

Using the same strategy, one may verify that the eq. (5.15) holds also for the following linear relations (cf. with section 3):

$$
\begin{aligned}
f_{0}+\tilde{f}_{0} & =-2 f^{\prime \prime \prime} \\
f^{\prime}+\tilde{f}^{\prime} & =2 \hat{f} \\
f^{\prime}-\tilde{f}^{\prime} & =-\sqrt{2}\left(f-\tilde{f}-f_{0}+\tilde{f}_{0}\right) .
\end{aligned}
$$

The relation

$$
\sqrt{2} d=2 c-2 \omega+\sqrt{2} \omega^{\prime}+\sqrt{2}\left(f^{\prime}-\tilde{f}^{\prime}\right)-\left(f_{0}-\tilde{f}_{0}\right)
$$

is more complicated. Using the identity $\Gamma_{36}-\Gamma_{45}+\Gamma_{63}-\Gamma_{54}=0$, we may rewrite eq. (B.22) in the following form:

$$
\begin{gathered}
\frac{1}{\sqrt{2}}\left(\Gamma_{12}+\Gamma_{21}\right)-2 \Gamma_{11}+\left(\Gamma_{13}+\Gamma_{31}\right)-\frac{1}{\sqrt{2}}\left(\Gamma_{14}+\Gamma_{41}\right) \\
-\frac{1}{\sqrt{2}}\left(\Gamma_{34}+\Gamma_{43}-\Gamma_{56}+\Gamma_{65}\right)+a\left(\Gamma_{36}-\Gamma_{45}+\Gamma_{63}-\Gamma_{54}\right)=0,
\end{gathered}
$$

where $a$ is arbitrary. Reading off the coefficients $d_{i j}$ from the above equation, one may verify by direct calculations that eq. (5.15) holds, if the choice $a=\sqrt{2}$ is made. 
We have further checked that the remaining identities

$$
\begin{aligned}
c^{\prime} & =c+\frac{d}{\sqrt{2}}-f^{\prime \prime \prime}-2 f^{\prime \prime}-2 \sqrt{2} \hat{f}-3 \omega+\frac{3}{\sqrt{2}} \omega^{\prime}, \\
\nu & =\sqrt{2} \omega+\hat{f}+\sqrt{2} f^{\prime \prime}, \\
\nu^{\prime} & =\sqrt{2} \omega^{\prime}-\sqrt{2} \hat{f}-f^{\prime \prime \prime},
\end{aligned}
$$

can be treated in a similar fashion. Adding the term $a\left(\Gamma_{36}-\Gamma_{45}+\Gamma_{63}-\Gamma_{54}\right)$ to the pertinent linear relations for the four-point functions, it is shown that the constant $a$ can be always adjusted so that the eq. (5.15) is fulfilled.

In section 3 more linear relations are displayed, which correspond to the transitions involving the states not affected by mixing. It is a straightforward task to verify that the same arguments can be applied in this case as well.

Open Access. This article is distributed under the terms of the Creative Commons Attribution License (CC-BY 4.0), which permits any use, distribution and reproduction in any medium, provided the original author(s) and source are credited.

\section{References}

[1] SCALAR collaboration, T. Kunihiro et al., Scalar mesons in lattice QCD, Phys. Rev. D 70 (2004) 034504 [hep-ph/0310312] [INSPIRE].

[2] UKQCD collaboration, C. McNeile and C. Michael, Properties of light scalar mesons from lattice QCD, Phys. Rev. D 74 (2006) 014508 [hep-lat/0604009] [INSPIRE].

[3] UKQCD collaboration, A. Hart, C. McNeile, C. Michael and J. Pickavance, A Lattice study of the masses of singlet 0++ mesons, Phys. Rev. D 74 (2006) 114504 [hep-lat/0608026] [INSPIRE].

[4] S. Prelovsek and D. Mohler, A Lattice study of light scalar tetraquarks, Phys. Rev. D 79 (2009) 014503 [arXiv:0810.1759] [INSPIRE].

[5] S. Prelovsek, T. Draper, C.B. Lang, M. Limmer, K.-F. Liu et al., Lattice study of light scalar tetraquarks with I=0,2,1/2,3/2: Are $\sigma$ and $\kappa$ tetraquarks?, Phys. Rev. D 82 (2010) 094507 [arXiv: 1005.0948] [INSPIRE].

[6] C. Alexandrou, J.O. Daldrop, M. Dalla Brida, M. Gravina, L. Scorzato et al., Lattice investigation of the scalar mesons $a_{0}(980)$ and $\kappa$ using four-quark operators, JHEP 04 (2013) 137 [arXiv:1212.1418] [INSPIRE].

[7] European Twisted Mass collaboration, M. Wagner et al., Scalar mesons and tetraquarks from twisted mass lattice QCD, Acta Phys. Polon. Supp. 6 (2013) 847 [arXiv:1302.3389] [INSPIRE].

[8] M. Lüscher, Two particle states on a torus and their relation to the scattering matrix, Nucl. Phys. B 354 (1991) 531 [inSPIRE].

[9] V. Bernard, M. Lage, U.-G. Meißner and A. Rusetsky, Scalar mesons in a finite volume, JHEP 01 (2011) 019 [arXiv: 1010.6018] [InSPIRE].

[10] M. Döring, U.-G. Meißner, E. Oset and A. Rusetsky, Unitarized Chiral Perturbation Theory in a finite volume: Scalar meson sector, Eur. Phys. J. A 47 (2011) 139 [arXiv:1107.3988] [INSPIRE]. 
[11] M. Döring, U. Meißner, E. Oset and A. Rusetsky, Scalar mesons moving in a finite volume and the role of partial wave mixing, Eur. Phys. J. A 48 (2012) 114 [arXiv:1205.4838] [INSPIRE].

[12] R.L. Jaffe, Multi-Quark Hadrons. 1. The Phenomenology of (2 Quark 2 anti-Quark) Mesons, Phys. Rev. D 15 (1977) 267 [INSPIRE].

[13] D. Black, A.H. Fariborz, F. Sannino and J. Schechter, Putative light scalar nonet, Phys. Rev. D 59 (1999) 074026 [hep-ph/9808415] [INSPIRE].

[14] N. Achasov and A. Kiselev, The New analysis of the KLOE data on the $\phi \rightarrow \eta \pi^{0} \gamma$ decay, Phys. Rev. D 68 (2003) 014006 [hep-ph/0212153] [INSPIRE].

[15] J. Pelaez, Light scalars as tetraquarks or two-meson states from large- $N_{c}$ and unitarized chiral perturbation theory, Mod. Phys. Lett. A 19 (2004) 2879 [hep-ph/0411107] [INSPIRE].

[16] J.D. Weinstein and N. Isgur, Do Multi-Quark Hadrons Exist?, Phys. Rev. Lett. 48 (1982) 659 [INSPIRE].

[17] J. Oller and E. Oset, Chiral symmetry amplitudes in the $S$ wave isoscalar and isovector channels and the sigma, f0(980), a0(980) scalar mesons, Nucl. Phys. A 620 (1997) 438 [Erratum ibid. A 652 (1999) 407] [hep-ph/9702314] [INSPIRE].

[18] J. Oller, E. Oset and J. Pelaez, Nonperturbative approach to effective chiral Lagrangians and meson interactions, Phys. Rev. Lett. 80 (1998) 3452 [hep-ph/9803242] [INSPIRE].

[19] J. Oller, E. Oset and J. Pelaez, Meson meson interaction in a nonperturbative chiral approach, Phys. Rev. D 59 (1999) 074001 [Erratum ibid. D 60 (1999) 099906] [hep-ph/9804209] [INSPIRE].

[20] J. Oller and E. Oset, $N / D$ description of two meson amplitudes and chiral symmetry, Phys. Rev. D 60 (1999) 074023 [hep-ph/9809337] [INSPIRE].

[21] J. Oller, E. Oset and J. Pelaez, Meson meson interaction in a nonperturbative chiral approach, Phys. Rev. D 59 (1999) 074001 [Erratum ibid. D 60 (1999) 099906] [hep-ph/9804209] [INSPIRE].

[22] S. Peris, M. Perrottet and E. de Rafael, Matching long and short distances in large- $N_{c} Q C D$, JHEP 05 (1998) 011 [hep-ph/9805442] [INSPIRE].

[23] V. Elias, A. Fariborz, F. Shi and T.G. Steele, QCD sum rule consistency of lowest lying $q \bar{q}$ scalar resonances, Nucl. Phys. A 633 (1998) 279 [hep-ph/9801415] [INSPIRE].

[24] G. Janssen, B. Pearce, K. Holinde and J. Speth, On the structure of the scalar mesons fo (975) and a0 (980), Phys. Rev. D 52 (1995) 2690 [nucl-th/9411021] [INSPIRE].

[25] S. Weinberg, Elementary particle theory of composite particles, Phys. Rev. 130 (1963) 776 [INSPIRE].

[26] S. Weinberg, Quasiparticles and the Born Series, Phys. Rev. 131 (1963) 440 [InSPIRE].

[27] S. Weinberg, Evidence That the Deuteron Is Not an Elementary Particle, Phys. Rev. B 137 (1965) 672.

[28] D. Morgan, Pole counting and resonance classification, Nucl. Phys. A 543 (1992) 632 [INSPIRE].

[29] N.A. Tornqvist, How to parametrize an $S$ wave resonance and how to identify two hadron composites, Phys. Rev. D 51 (1995) 5312 [hep-ph/9403234] [INSPIRE]. 
[30] D. Morgan and M. Pennington, fo $\left(S^{*}\right)$ : Molecule or quark state?, Phys. Lett. B 258 (1991) 444 [Erratum ibid. B 269 (1991) 477] [INSPIRE].

[31] D. Morgan and M. Pennington, New data on the $K \bar{K}$ threshold region and the nature of the fo $\left(S^{*}\right)$, Phys. Rev. D 48 (1993) 1185 [inSPIRE].

[32] V. Baru, J. Haidenbauer, C. Hanhart, Y. Kalashnikova and A.E. Kudryavtsev, Evidence that the $a(0)(980)$ and $f(0)(980)$ are not elementary particles, Phys. Lett. B 586 (2004) 53 [hep-ph/0308129] [INSPIRE].

[33] V. Baru, J. Haidenbauer, C. Hanhart, A.E. Kudryavtsev and U.-G. Meißner, Flatte-like distributions and the $a(0)(980) / f(0)(980)$ mesons, Eur. Phys. J. A 23 (2005) 523 [nucl-th/0410099] [INSPIRE].

[34] C. Hanhart, Towards an understanding of the light scalar mesons, Eur. Phys. J. A 31 (2007) 543 [hep-ph/0609136] [INSPIRE].

[35] P.F. Bedaque, Aharonov-Bohm effect and nucleon nucleon phase shifts on the lattice, Phys. Lett. B 593 (2004) 82 [nucl-th/0402051] [INSPIRE].

[36] G. de Divitiis, R. Petronzio and N. Tantalo, On the discretization of physical momenta in lattice QCD, Phys. Lett. B 595 (2004) 408 [hep-lat/0405002] [INSPIRE].

[37] G.M. de Divitiis and N. Tantalo, Non leptonic two-body decay amplitudes from finite volume calculations, hep-lat/0409154 [INSPIRE].

[38] C. Sachrajda and G. Villadoro, Twisted boundary conditions in lattice simulations, Phys. Lett. B 609 (2005) 73 [hep-lat/0411033] [INSPIRE].

[39] P.F. Bedaque and J.-W. Chen, Twisted valence quarks and hadron interactions on the lattice, Phys. Lett. B 616 (2005) 208 [hep-lat/0412023] [INSPIRE].

[40] S. Ozaki and S. Sasaki, Lüscher's finite size method with twisted boundary conditions: an application to $J / \psi-\phi$ system to search for narrow resonance, Phys. Rev. D 87 (2013) 014506 [arXiv:1211.5512] [INSPIRE].

[41] S. Beane, P. Bedaque, A. Parreno and M. Savage, Exploring hyperons and hypernuclei with lattice QCD, Nucl. Phys. A 747 (2005) 55 [nucl-th/0311027] [INSPIRE].

[42] V. Bernard, M. Lage, U.-G. Meißner and A. Rusetsky, Resonance properties from the finite-volume energy spectrum, JHEP 08 (2008) 024 [arXiv:0806.4495] [INSPIRE].

[43] V. Bernard, D. Hoja, U. Meißner and A. Rusetsky, Matrix elements of unstable states, JHEP 09 (2012) 023 [arXiv: 1205.4642] [INSPIRE].

[44] S.R. Sharpe and N. Shoresh, Partially quenched chiral perturbation theory without Phi0, Phys. Rev. D 64 (2001) 114510 [hep-lat/0108003] [INSPIRE].

[45] C. Bernard and M. Golterman, On the foundations of partially quenched chiral perturbation theory, Phys. Rev. D 88 (2013) 014004 [arXiv: 1304.1948] [INSPIRE].

[46] J. Gasser, V. Lyubovitskij and A. Rusetsky, Hadronic atoms in QCD + QED, Phys. Rept. 456 (2008) 167 [arXiv:0711.3522] [INSPIRE].

[47] J. Gasser, B. Kubis and A. Rusetsky, Cusps in $K \rightarrow 3 \pi$ decays: a theoretical framework, Nucl. Phys. B 850 (2011) 96 [arXiv:1103.4273] [InSPIRE].

[48] H. Feshbach, Unified theory of nuclear reactions, Annals Phys. 5 (1958) 357 [InSPIRE].

[49] H. Feshbach, A Unified theory of nuclear reactions. 2., Annals Phys. 19 (1962) 287 [InSPIRE]. 
[50] K. Rummukainen and S.A. Gottlieb, Resonance scattering phase shifts on a nonrest frame lattice, Nucl. Phys. B 450 (1995) 397 [hep-lat/9503028] [INSPIRE].

[51] M. Göckeler, R. Horsley, M. Lage, U.-G. Meißner, P. Rakow et al., Scattering phases for meson and baryon resonances on general moving-frame lattices, Phys. Rev. D 86 (2012) 094513 [arXiv: 1206 .4141] [INSPIRE].

[52] L. Leskovec and S. Prelovsek, Scattering phase shifts for two particles of different mass and non-zero total momentum in lattice QCD, Phys. Rev. D 85 (2012) 114507 [arXiv: 1202.2145] [INSPIRE].

[53] N. Li and C. Liu, Generalized Lüscher Formula in Multi-channel Baryon-Meson Scattering, Phys. Rev. D 87 (2013) 014502 [arXiv:1209.2201] [INSPIRE].

[54] J. Bijnens and N. Danielsson, The Eta mass and NNLO three-flavor partially quenched chiral perturbation theory, Phys. Rev. D 74 (2006) 054503 [hep-lat/0606017] [INSPIRE]. 